\title{
Review Article \\ Theoretical Studies of Dynamic Interactions in Excited States of Hydrogen-Bonded Systems
}

\author{
Marek J. Wójcik, Marek Boczar, and Lukasz Boda \\ Faculty of Chemistry, Jagiellonian University, Ingardena 3, Kraków 30-060, Poland \\ Correspondence should be addressed to Marek J. Wójcik, wojcik@chemia.uj.edu.pl \\ Received 1 March 2012; Accepted 19 May 2012 \\ Academic Editor: Paul Blaise
}

Copyright ( $) 2012$ Marek J. Wójcik et al. This is an open access article distributed under the Creative Commons Attribution License, which permits unrestricted use, distribution, and reproduction in any medium, provided the original work is properly cited.

\begin{abstract}
Theoretical model for vibrational interactions in the hydrogen-bonded benzoic acid dimer is presented. The model takes into account anharmonic-type couplings between the high-frequency $\mathrm{O}-\mathrm{H}$ and the low-frequency $\mathrm{O} \cdots \mathrm{O}$ stretching vibrations in two hydrogen bonds, resonance interactions between two hydrogen bonds in the dimer, and Fermi resonance between the $\mathrm{O}-\mathrm{H}$ stretching fundamental and the first overtone of the $\mathrm{O}-\mathrm{H}$ in-plane bending vibrations. The model is used for theoretical simulation of the $\mathrm{O}-\mathrm{H}$ stretching IR absorption bands of benzoic acid dimers in the gas phase in the first excited singlet state. Ab initio CIS and CIS(D)/CIS/6-311++G(d,p) calculations have been carried out in the Â state of tropolone. The grids of potential energy surfaces along the coordinates of the tunneling vibration and the low-frequency coupled vibration have been calculated. Two-dimensional model potentials have been fitted to the calculated potential energy surfaces. The tunneling splittings for vibrationally excited states have been calculated and compared with the available experimental data. The model potential energy surfaces give good estimation of the tunneling splittings in the vibrationally ground and excited states of tropolone, and explain monotonic decrease in tunneling splittings with the excitation of low-frequency out-of-plane modes and increase of the tunneling splittings with the excitation of low-frequency planar modes.
\end{abstract}

\section{Introduction}

There is recently a considerable interest in studies of hydrogen-bonded carboxylic acid dimers. The main reason comes from the fact that they constitute good models to study systems with two interacting intermolecular hydrogen bonds. Of special interest is the process of double proton tunneling along hydrogen bonds in both the ground and excited electronic states.

Electronic spectroscopy of hydrogen-bonded dimers has recently received considerable attention due to significant development and the widespread use of supersonic free jet techniques. Many investigators still use classical spectroscopic techniques, such as the vibrational spectroscopy, to study hydrogen-bonded complexes in their ground electronic states.

It is well known that vibrational spectra provide physical information on the dynamics of hydrogen bonds. The most prominent spectral changes resulting from $\mathrm{H}$ bond formation occur in the IR spectra, especially in the region of the $\mathrm{X}-\mathrm{H}$ stretching bands $\left(v_{s}\right)$ : decrease in the frequency of the $v_{s}$ stretching mode, increase of its intensity, broadening of the bands and appearance of a complex fine structure. These spectacular features of the infrared absorption band of the $v_{s}$ mode have been a subject of several theoretical studies [111 .

An anharmonic-type coupling between the high-frequency $\mathrm{X}-\mathrm{H}$ stretching and the low-frequency $\mathrm{X} \cdots \mathrm{Y}$ hydrogen bond stretching vibration, described in a quantum mechanical way by Maréchal and Witkowski [1] is an important mechanism responsible for the unique structure of $v_{s}$ bands of hydrogen-bonded systems. Another important mechanism influencing the fine structure of $v_{s}$ band is Fermi resonance. Theoretical quantum mechanical model treating this mechanism was proposed by Witkowski and Wójcik [3] 
for a single hydrogen bond and by Wójcik [4] for hydrogenbonded dimers.

Benzoic acid is the simplest aromatic carboxylic acid and is also one of the most important acids in chemistry. The electronic spectra of benzoic acid have been a subject of extensive studies for a long time. Ito et al. [12] investigated the effect of dimerization on the UV absorption spectrum. Baba and Kitamura [13] examined the emission properties of the monomer and the dimer in glass solution. Baum and McClure, in series of papers, examined the absorption and emission spectra of benzoic acid dimers in single crystals of benzene $[14,15]$ as well as in hydrocarbon glasses $[16,17]$. Later, Poeltl and McVey have reported for the first time the laser induced fluorescence excitation spectrum [18] and next the fluorescence emission spectra [19] of jet-cooled benzoic acid dimers. Tomioka et al. [20] studied the correlation between the frequencies of intermolecular hydrogen bond vibrations between the fluorescence excitation and dispersed fluorescence spectra and concluded that potentials for such vibrations are affected very little upon electronic excitation. Significant discovery was made by Remmers and et al. [21] . On the basis of their high resolution ultraviolet rotationally resolved excitation spectrum of benzoic acid dimer, they have demonstrated convincingly that the linear and planar $\left(\mathrm{C}_{2 h}\right.$ symmetry) ground state geometry of the dimer is slightly in-plane bent $\left(\mathrm{C}_{s}\right.$ symmetry) upon electronic excitation. Recently Nandi and Chakraborty [22] have reinvestigated the laser-induced dispersed fluorescence spectra and analyzed vibronic mode mixing in benzoic acid dimer with aid of DFT calculations. Among jet spectroscopic techniques there is increasing use of double resonance methods, which allow measure, for example, IR absorption of electronically excited species.

In recent years, infrared spectra of the $\mathrm{O}-\mathrm{H}$ stretch region of benzoic acid dimer have been studied in both the ground and excited electronic state by Florio and et al. [23] using the FDIR (fluorescence-dip infrared) IR-UV double resonance method in supersonic jet. The authors have also computed theoretical IR spectrum of benzoic acid dimer using anharmonic constants [23, 24]. Benzoic acid has been also studied in the ground state by traditional spectroscopic techniques. Infrared spectra of hydrogen-bonded benzoic acid crystals have been recorded and interpreted theoretically by Flakus et al. [25-29] and more recently by Boczar et al. [30] The present work constitutes a development of this study and is also a continuation of our recent spectroscopic and theoretical studies of vibrational spectra of hydrogenbonded 1-methylthymine [31], acetic acid [32], and salicylic acid [33]. Theoretical studies on different hydrogen-bonded chemical and biochemical systems in excited electronic states have been performed in recent years [34-37].

The motion of protons in hydrogen bonds causes great number of interesting physical effects. Quantum effects, such as the proton tunneling phenomenon, and strong interactions with vibrating surrounding atoms in hydrogen bonds are of special interest. The importance of proton tunneling in chemical and biological systems is well known, for example, for the DNA base pairing, as discussed by Löwdin [38]. The phenomenon of potential barrier penetration plays an important role in many branches of physics: quantum field theory, fission of atomic nuclei, scanning tunneling microscopy, and solid state physics [39]. In recent decade appeared several theoretical studies of proton tunneling in different systems [40-49].

Theoretical studies of proton tunneling require the knowledge of multidimensional potential energy surfaces which are difficult to obtain from $a b$ initio calculations, especially for electronically excited states. Tropolone with its intramolecular hydrogen bond is a model substance for studying tunneling process in the ground as well as in the excited electronic state [50-59]. The geometry of tropolone is presented in Figure 4. Multidimensional proton tunneling in tropolone has been theoretically studied by Vener et al. [56] using adiabatic separation of variables. Smedarchina et al. [57] used instanton approach to account for tunneling splittings. Takada and Nakamura [58] studied model potentials. On the base of $a b$ initio calculations they proposed model potential energy surfaces (PES) for electronically ground $\tilde{X}$ state of tropolone and employed it to analyze dynamics of proton tunneling. They were however unable to perform similar calculations for the excited $\tilde{A}$ state. In this paper we report results of high accuracy $a b$ initio calculations of the potential energy surface in the excited $\tilde{A}$ state of tropolone. We fit two-dimensional analytical model potentials to these surfaces and by solving the two-dimensional vibrational problems, we interpret observed splittings and their dependence on vibrational excitations in the laser fluorescence excitation spectra of jet-cooled tropolone [53, 54]. This work constitutes improvement of previous approach of Wójcik et al. [59] to multidimensional proton tunneling in the excited state of tropolone.

This review presents results published in $[60,61]$. In Section 2 we present theoretical interpretation of vibrational interactions in hydrogen bonds in benzoic acid dimer in the first excited electronic state and interpret its experimental FDIR spectrum recorded by Florio et al. [23]. The theoretical model describing these interactions is proposed and used to simulate the fine structure of the $\mathrm{O}-\mathrm{H}$ stretching IR absorption band in the $S_{1}$ electronic state. In addition quantum mechanical $a b$ initio calculations have been made in order to obtain the excited state structure of benzoic acid dimer and its vibrational frequencies. In Section 3 we present the results of quantum chemical calculations for the $\tilde{A}$ state of tropolone. We discuss two-dimensional potential models of the tunneling and interpret experimentally observed tunneling splittings $[53,54]$. Concluding remarks are given at the end of each section.

\section{Theoretical Interpretation of Vibrational Interactions in Hydrogen Bonds in Benzoic Acid Dimer in the First Excited Singlet State $S_{1}$}

2.1. Quantum Chemical Calculations. All calculations have been carried out using the Gaussian 03 package [62]. The vertical singlet state energies were obtained by the $a b$ initio single excitation configuration-interaction calculations at 


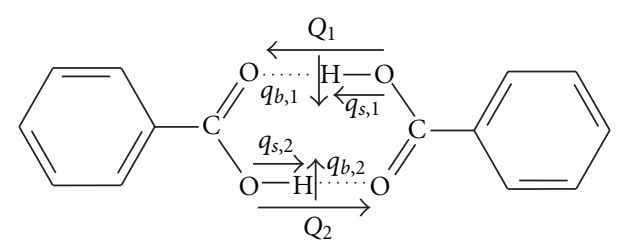

FIGURE 1: Oscillatory motions in the hydrogen bonds in the benzoic acid dimer considered in the models.

the CIS [63] and CIS(D) [64, 65] levels with the 6$311++\mathrm{G}(\mathrm{d}, \mathrm{p})$ basis set. The calculations were performed for the five lowest singlet excited states of benzoic acid dimer starting from the $\mathrm{C}_{2 h}$ ground state geometry optimized at B3LYP/6-311++G(d,p) level. The RHF/6-311++G(d,p) population analysis was also performed for the ground state geometry in order to examine the orbitals involved in proper electronic excitations.

In the first excited singlet state $\left(S_{1}\right)$ the geometry of benzoic acid dimer was optimized and the vibrational frequencies were computed at the CIS/6-311++G(d,p) level. To ensure reliable frequencies of low-frequency vibrational modes (with very small force constants), especially intermolecular, in the present calculations we used the tight convergence criteria.

2.2. Theoretical Model. We make the following physical assumptions in the model of the $v_{s}$ IR bandshapes of the benzoic acid dimer in the gas phase in its electronic ground $S_{0}$ and the first excited $S_{1}$ state. This model will be use for quantitative calculations of the spectra.

(1) In our model the basic physical mechanism responsible for the energy and intensity distributions within the IR O-H stretching absorption band is an anharmonic-type coupling between the high-frequency $\mathrm{O}-\mathrm{H}$ stretching vibration and the lowfrequency hydrogen bond $\mathrm{O} \cdots \mathrm{O}$ stretching vibration $\left(v_{\sigma}\right)$ in each hydrogen bond. Since the $v_{s}$ oscillatory motion is at least an order of magnitude faster than the oscillatory motion $v_{\sigma}$, the vibrational wavefunction for these modes is represented in crude adiabatic approximation. This approximation is true only for weak and medium strong hydrogen bonds. The high-frequency $v_{s}$ vibration determines potential for the low-frequency $v_{\sigma}$ vibration in each hydrogen bond. The $v_{s}$ and $v_{\sigma}$ vibrations are assumed to be harmonic.

(2) When $v_{s}$ vibration is excited, there is shift of equilibrium position of the potential energy for $\nu_{\sigma}$ vibration (linear distortion). We do not consider change of force constant after excitation.

(3) In the ground electronic state $\left(S_{0}\right)$ the benzoic acid dimer has $C_{2 h}$ symmetry, therefore in the case of two equivalent intermolecular hydrogen bonds, present in the dimer, a nonadiabatic resonance interaction (Davydov coupling) is considered in the degenerate excited vibrational state of the $v_{s}$ vibrations. This effect is a vibrational analogue of the vibronic coupling, such as the pseudo-Jahn-Teller effect, occurring in the electronic spectra of symmetric dimers [64]. From the experimental data it has been concluded that in the first excited electronic state benzoic acid dimer is in-plane bent as an effect of localised electronic excitation on one moiety of the dimer $[14,18,19,21]$.

(4) In our model we also consider Fermi resonance between the $\mathrm{O}-\mathrm{H}$ stretching fundamental and the first overtone of the $\mathrm{O}-\mathrm{H}$ in-plane bending $\left(\nu_{b}\right)$ in each hydrogen bond in the dimer.

2.2.1. Vibrational Hamiltonians for The Dimer. Let us consider a planar cyclic dimer of benzoic acid, presented in Figure 1. In the ground electronic state it has $C_{2 h}$ symmetry with two hydrogen bonds, linking two moieties of the dimer, related by the symmetry operator $\hat{C}_{2}$ corresponding to twofold symmetry axis. Theoretical model of such dimer with Fermi resonance, presented below, has been developed by Wójcik [4].

We denote by $q_{s, i}, q_{b, i}$, and $Q_{i}(i=1,2)$ the coordinates of the $\mathrm{O}-\mathrm{H}$ stretching, $\mathrm{O}-\mathrm{H}$ in-plane bending, and $\mathrm{O} \cdots \mathrm{O}$ hydrogen bond stretching vibrations in the first or second hydrogen bond (Figure 1). The corresponding frequencies are denoted by $\omega_{s}, \omega_{b}, \Omega$.

The vibrational Hamiltonian $\hat{H}$ of the dimer has the form:

$$
\begin{aligned}
\hat{H}= & \widehat{T}\left(Q_{1}\right)+\widehat{T}\left(Q_{2}\right)+\hat{h}_{s, 1}+\hat{h}_{s, 2}+\hat{h}_{b, 1}+\hat{h}_{b, 2} \\
& +V_{\mathrm{ah}, 1}+V_{\mathrm{ah}, 2}+V_{\mathrm{res}},
\end{aligned}
$$

where $\widehat{T}\left(Q_{i}\right)$ are the kinetic energy operators of the lowfrequency $\mathrm{O} \cdots$ O vibrations; $\hat{h}_{s, i}=\hat{T}\left(q_{s, i}\right)+U\left(q_{s, i}, Q_{i}\right)$ the vibrational Hamiltonians of the high frequency $\mathrm{O}-\mathrm{H}$ stretching vibrations; $\hat{h}_{b, i}=\widehat{T}\left(q_{b, i}\right)+U\left(q_{b, i}\right)$ vibrational Hamiltonians of the high-frequency $\mathrm{O}-\mathrm{H}$ in-plane bending vibrations; $V_{\mathrm{ah}, i}$ anharmonic coupling terms between the $\mathrm{O}-\mathrm{H}$ stretching and $\mathrm{O}-\mathrm{H}$ in-plane bending vibrations; $V_{\text {res }}\left(q_{s, 1}, q_{s, 2}\right)$ is a resonance coupling between two equivalent hydrogen bonds. $U$ denotes the potential energy.

The total vibrational wavefunction $\Psi_{j^{\prime}}^{+}$describing the first excited state of the $v_{s}$ vibration takes a four-component form:

$$
\begin{aligned}
\Psi_{m}^{+}= & \alpha_{1} \varphi_{s, 1}^{+} \varphi_{b, 1} \varphi_{s, 2} \varphi_{b, 2}+\beta_{1} \varphi_{s, 1} \varphi_{b, 1}^{+} \varphi_{s, 2} \varphi_{b, 2} \\
& +\alpha_{2} \varphi_{s, 1} \varphi_{b, 1} \varphi_{s, 2}^{+} \varphi_{b, 2}+\beta_{2} \varphi_{s, 1} \varphi_{b, 1} \varphi_{s, 2} \varphi_{b, 2}^{+}
\end{aligned}
$$

where $\varphi_{s, i}\left(q_{s, i}, Q_{i}\right)$ are the eigenfunctions of the Hamiltonians $\hat{h}_{s, i} ; \varphi_{b, i}\left(q_{b, i}\right)$ the eigenfunctions of the Hamiltonians $\hat{h}_{b, i}$; and $\alpha_{i}(Q), \beta_{i}(Q)$ are the wavefunctions of the hydrogen bond vibrations $\nu_{\sigma}$, not yet determined.

To determine these wavefunctions and the total vibrational energy we use the variational principle [66]:

$$
\delta\left\langle\Psi_{m}^{+}|\hat{H}| \Psi_{m}^{+}\right\rangle=E_{m} \delta\left\langle\Psi_{m}^{+} \mid \Psi_{m}^{+}\right\rangle
$$


applied to the Schrödinger equation with the Hamiltonian (1) and the wavefunction (2). With the crude adiabatic approximation assumed for the $v_{s}$ and $v_{\sigma}$ vibrations
$\left(\left[\widehat{T}\left(Q_{i}\right), \chi_{s, i}\right]=0\right)$, the effective Hamiltonian $\hat{\mathbf{H}}^{\text {eff }}$ for the lowfrequency vibrations $v_{\sigma}$ takes the four-dimensional matrix form:

$$
\hat{\mathbf{H}}^{\text {eff }} \boldsymbol{Q}=\left[\begin{array}{c}
\hat{T}\left(Q_{1}\right)+\widehat{T}\left(Q_{2}\right)+\varepsilon_{s, 1}^{+}\left(Q_{1}\right)+\varepsilon_{s, 2}\left(Q_{2}\right)+\varepsilon_{b, 1}+\varepsilon_{b, 2}, V_{\text {int }}^{\text {ah }}, V_{\text {int }}^{\text {res }}, 0 \\
V_{\text {iht }}^{\text {ah }}, \widehat{T}\left(Q_{1}\right)+\widehat{T}\left(Q_{2}\right)+\varepsilon_{s, 1}\left(Q_{1}\right)+\varepsilon_{s, 2}\left(Q_{2}\right)+\varepsilon_{b, 1}^{+}+\varepsilon_{b, 2}, 0,0 \\
V_{\text {int }}^{\text {res }}, 0, \widehat{T}\left(Q_{1}\right)+\widehat{T}\left(Q_{2}\right)+\varepsilon_{s, 1}\left(Q_{1}\right)+\varepsilon_{s, 2}^{+}\left(Q_{2}\right)+\varepsilon_{b, 1}+\varepsilon_{b, 2}, V_{\text {int }}^{\text {ah }} \\
0,0, V_{\text {int }}^{\text {ah }} \widehat{T}\left(Q_{1}\right)+\widehat{T}\left(Q_{2}\right)+\varepsilon_{s, 1}\left(Q_{1}\right)+\varepsilon_{s, 2}\left(Q_{2}\right)+\varepsilon_{b, 1}+\varepsilon_{b, 2}^{+}
\end{array}\right],
$$

where $\varepsilon_{s, i}\left(Q_{i}\right)$ are the eigenvalues of the Hamiltonians $\hat{h}_{s, i}, \varepsilon_{b, i}$ the eigenvalues of the Hamiltonians $\hat{h}_{b, i}, V_{\text {int }}^{\text {ah }}$ the matrix element of the anharmonic coupling between excited states of $v_{s}$ and $v_{b}$, and $V_{\mathrm{int}}^{\text {res }}$ is the matrix element of resonance interaction (vibrational analogue of the exchange integral). They are defined as:

$$
\begin{aligned}
V_{\text {int }}^{\mathrm{ah}} & =\left\langle\varphi_{s, 1}^{+} \varphi_{b, 1} \varphi_{s, 2} \varphi_{b, 2}\left|V_{\mathrm{ah}, 1}\right| \varphi_{s, 1} \varphi_{b, 1}^{+} \varphi_{s, 2} \varphi_{b, 2}\right\rangle_{q_{s}, q_{b}} \\
& =\left\langle\varphi_{s, 1} \varphi_{b, 1} \varphi_{s, 2}^{+} \varphi_{b, 2}\left|V_{\mathrm{ah}, 2}\right| \varphi_{s, 1} \varphi_{b, 1} \varphi_{s, 2} \varphi_{b, 2}^{+}\right\rangle_{q_{s}, q_{b}}, \\
V_{\mathrm{int}}^{\mathrm{res}} & =\left\langle\varphi_{s, 1}^{+} \varphi_{b, 1} \varphi_{s, 2} \varphi_{b, 2}\left|V_{\mathrm{res}}\right| \varphi_{s, 1} \varphi_{b, 1} \varphi_{s, 2}^{+} \varphi_{b, 2}\right\rangle_{q_{s}, q_{b}} .
\end{aligned}
$$

The energies $\varepsilon_{s, i}\left(Q_{i}\right)$ of the high-frequency $\mathrm{O}-\mathrm{H}$ stretching vibrations in individual hydrogen bonds determine effective potential for the low-frequency hydrogen bond vibrations. We assume that these potentials are harmonic with the same force constant in the ground and excited states of the $\mathrm{O}-\mathrm{H}$ stretching vibrations:

$$
\varepsilon_{s, i}=\frac{1}{2} K Q_{i}^{2}, \quad \varepsilon_{s, i}^{+}=R+L Q_{i}+\frac{1}{2} K Q_{i}^{2},
$$

where $R$ is the vertical excitation energy, $L$ the linear distortion parameter, and $K$ is the force constant.

Introducing dimensionless quantities:

$$
\begin{gathered}
q_{i}=Q_{i}\left(\frac{M \Omega}{\hbar}\right)^{1 / 2}, \quad p_{i}=\frac{P_{i}}{(\hbar M \Omega)^{1 / 2}}, \\
b=\frac{L}{\left(\hbar M \Omega^{3}\right)^{1 / 2}}, \\
r=\frac{R}{\hbar \Omega}, \quad r^{\prime}=\frac{R^{\prime}}{\hbar \Omega}, \\
V^{\text {ah }}=\frac{V_{\text {int }}^{\text {ah }}}{\hbar \Omega}, \quad V^{\text {res }}=\frac{V_{\text {int }}^{\text {res }}}{\hbar \Omega},
\end{gathered}
$$

where $\Omega$ is the angular frequency and $M$ the reduced mass for the $v_{\sigma}$ vibration we can rewrite the Hamiltonian (4) in the following form:

$$
\begin{aligned}
\hat{\mathbf{h}}^{\mathrm{eff}}= & \frac{\hat{\mathbf{H}}^{\mathrm{eff}}}{\hbar \Omega} \\
& =\left[\begin{array}{c}
\frac{1}{2} \sum_{i=1}^{2}\left(p_{i}^{2}+q_{i}^{2}\right)+b q_{1}+r, V^{\mathrm{ah}}, V^{\mathrm{res}}, 0 \\
V^{\mathrm{ah}}, \frac{1}{2} \sum_{i=1}^{2}\left(p_{i}^{2}+q_{i}^{2}\right)+r^{\prime}, 0,0 \\
V^{\mathrm{res}}, 0, \frac{1}{2} \sum_{i=1}^{2}\left(p_{i}^{2}+q_{i}^{2}\right)+b q_{2}+r, V^{\mathrm{ah}} \\
0,0, V^{\mathrm{ah}}, \frac{1}{2} \sum_{i=1}^{2}\left(p_{i}^{2}+q_{i}^{2}\right)+r^{\prime}
\end{array}\right] .
\end{aligned}
$$

Dimensionless parameters $r$ and $r^{\prime}$ describe vertical excitation energies to the first excited state of the $v_{s}$ vibration and to the first overtone of the $\nu_{b}$ vibration. For exact Fermi resonance $r=r^{\prime}$.

The four-dimensional matrix Hamiltonian (8) can be reduced to the two-dimensional Hamiltonians $\widehat{\mathbf{h}}^{+}$and $\widehat{\mathbf{h}}^{-}$ using the symmetry operator $\hat{C}_{2}$. The method of reduction was devised by Fulton and Gouterman [67]. The Hamiltonians $\widehat{\mathbf{h}}^{+}$and $\widehat{\mathbf{h}}^{-}$are given by the formula:

$$
\hat{\mathbf{h}}^{ \pm}=\left[\begin{array}{c}
\frac{1}{2} \sum_{i=1}^{2}\left(p_{i}^{2}+q_{i}^{2}\right)+b q_{1}+r+V^{\mathrm{res}} \widehat{C}_{2}, V^{\mathrm{ah}} \\
V^{\mathrm{ah}}, \frac{1}{2} \sum_{i=1}^{2}\left(p_{i}^{2}+q_{i}^{2}\right)+r^{\prime}
\end{array}\right] .
$$

Eigenfunctions of this Hamitonians have the spinor form:

$$
\left[\begin{array}{c}
\alpha_{ \pm}^{(m)} \\
\beta_{ \pm}^{(m)}
\end{array}\right]
$$

In the first excited singlet state benzoic acid dimer is in-plane bent $[14,19,21]$. Such symmetry lowering (from $C_{2 h}$ to $C_{s}$ ) causes that degeneracy is removed and Davydov coupling significantly decreases. Also two hydrogen bonds are no longer equivalent, thus the model parameters (linear distortion $b$, vertical excitation energies $r$ and $r^{\prime}$, and matrix elements describing Fermi resonance $V_{\text {int }}^{\text {ah }}$ must be different for two hydrogen bonds. Since benzoic acid dimer is no longer centrosymmetric in the $S_{1}$ state, the effective matrix Hamiltonian (8) cannot be reduced in this case to 
TABLE 1: Calculated wavelengths of electronic transitions to the three lowest allowed [1] $\left(\pi, \pi^{*}\right)$ states of benzoic acid dimer.

\begin{tabular}{lccc}
\hline Electronic state & Experiment $[16,18]$ & $\mathrm{CIS} / 6-311++\mathrm{G}(\mathrm{d}, \mathrm{p})$ & $\mathrm{CIS}(\mathrm{D}) / 6-311++\mathrm{G}(\mathrm{d}, \mathrm{p})$ \\
\hline $\mathrm{S}_{1}\left(\mathrm{~B}_{\mathrm{u}}\right)$ & $280 \mathrm{~nm}\left(35724 \mathrm{~cm}^{-1}\right)$ & $214 \mathrm{~nm}$ & $248 \mathrm{~nm}$ \\
$\mathrm{~S}_{2}\left(\mathrm{~B}_{\mathrm{u}}\right)$ & $230 \mathrm{~nm}$ & $211 \mathrm{~nm}$ & $204 \mathrm{~nm}$ \\
$\mathrm{~S}_{3}\left(\mathrm{~A}_{\mathrm{u}}\right)$ & $190 \mathrm{~nm}$ & $178 \mathrm{~nm}$ & $182 \mathrm{~nm}$ \\
\hline
\end{tabular}

two-dimensional Hamiltonians by the Fulton-Gouterman method and takes the following form:

$$
\widehat{\mathbf{h}}_{\left(\mathrm{S}_{1}\right)}^{\mathrm{eff}}=\left[\begin{array}{c}
\frac{1}{2} \sum_{i=1}^{2}\left(p_{i}^{2}+q_{i}^{2}\right)+b q_{1}+r_{1}, V_{1}^{\mathrm{ah}}, V^{\mathrm{res}}, 0 \\
V_{1}^{\mathrm{ah}}, \frac{1}{2} \sum_{i=1}^{2}\left(p_{i}^{2}+q_{i}^{2}\right)+r_{1}^{\prime}, 0,0 \\
V^{\mathrm{res}}, 0, \frac{1}{2} \sum_{i=1}^{2}\left(p_{i}^{2}+q_{i}^{2}\right)+b q_{2}+r_{2}, V_{2}^{\mathrm{ah}} \\
0,0, V_{2}^{\mathrm{ah}}, \frac{1}{2} \sum_{i=1}^{2}\left(p_{i}^{2}+q_{i}^{2}\right)+r_{2}^{\prime}
\end{array}\right] .
$$

Compared with (8), the model parameters in the Hamiltonian (10) have indices 1 or 2 to describe two hydrogen bonds, which are no longer equivalent in the excited electronic state of the benzoic acid dimer.

2.2.2. Intensities. IR intensities of the transitions from the ground state to the excited state of the $\mathrm{O}-\mathrm{H}$ stretching vibrations are given by the formula:

$$
I_{n m}=\left|\left\langle\Psi_{n}|\hat{\vec{\mu}}| \Psi_{m}^{+}\right\rangle\right|^{2} \exp \left[-\frac{E_{n}}{k T}\right],
$$

where $\Psi_{n}$ is the $n$th wavefunction of the ground vibrational state of the $v_{s}$ and $v_{b}$ vibrations, $\Psi_{m}^{+}$is the $m$ th wavefunction of the excited vibrational state, $\vec{\mu}$ is the dipole moment of the dimer, and $E_{n}$ is the vibrational energy of the ground vibrational state of the $v_{s}$ and $v_{b}$ vibrations.

The wavefunctions $\Psi_{m}^{+}$are given by (2) and the wavefunctions $\Psi_{n}$, in the adiabatic approximation, have the form:

$$
\Psi_{n}=\alpha_{0}^{(n)} \varphi_{s, 1} \varphi_{b, 1} \varphi_{s, 2} \varphi_{b, 2}
$$

Neglecting dependence of the dipole moment $\vec{\mu}$ on the coordinates $Q$ of the low-frequency hydrogen bond vibration, we obtain the intensity given as a combination of the FranckCondon integrals between the wavefunctions $\alpha_{0}^{(n)}$ and the symmetrical low-frequency eigenfunctions $\alpha_{ \pm}^{(m)}, \beta_{ \pm}^{(m)}$ of the Hamiltonians (9):

$$
\begin{aligned}
I_{n m}^{I R\left(S_{0}\right)}=\left|M_{0 s}\right| \mid\left\langle\alpha_{0}^{(n)}\left|1 \pm \hat{C}_{2}\right| \alpha_{ \pm}^{(m)}\right\rangle & \\
& +\left.\delta\left\langle\alpha_{0}^{(n)}\left|1 \pm \hat{C}_{2}\right| \beta_{ \pm}^{(m)}\right\rangle\right|^{2} \exp \left[-\frac{E_{n}}{k T}\right]
\end{aligned}
$$

where:

$$
M_{0 s}=\left\langle\varphi_{s, 1} \varphi_{b, 1} \varphi_{s, 2} \varphi_{b, 2}|\hat{\vec{\mu}}| \varphi_{s, 1}^{+} \varphi_{b, 1} \varphi_{s, 2} \varphi_{b, 2}\right\rangle_{q_{s}, q_{b}}=\text { const }
$$

and $\delta^{2}$ is the ratio of the intensities of the bending overtone to the fundamental stretching bands.

In the $S_{1}$ state of the dimer, due to nonequivalence of the two hydrogen bonds, the formula for intensities of IR transitions takes the form:

$$
\begin{gathered}
I_{n m}^{I R\left(S_{1}\right)} \sim \mid\left\langle\alpha_{0}^{(n)} \mid \alpha_{1}^{(m)}\right\rangle+\delta\left\langle\alpha_{0}^{(n)} \mid \beta_{1}^{(m)}\right\rangle+\left\langle\alpha_{0}^{(n)} \mid \alpha_{2}^{(m)}\right\rangle \\
+\left.\delta\left\langle\alpha_{0}^{(n)} \mid \beta_{2}^{(m)}\right\rangle\right|^{2} \exp \left[-\frac{E_{n}}{k T}\right] .
\end{gathered}
$$

To obtain this formula we used spinor eigenfunctions of the Hamiltonian (11):

$$
\left[\begin{array}{l}
\alpha_{1}^{(m)} \\
\beta_{1}^{(m)} \\
\alpha_{2}^{(m)} \\
\beta_{2}^{(m)}
\end{array}\right] .
$$

For simplicity we assumed in the formula (16) that transition moments (15) are the same for both hydrogen bonds in the dimer in the $S_{1}$ state.

2.3. Results and Discussion. The UV absorption spectrum of benzoic acid consists of three bands: A $(190 \mathrm{~nm})$, B $(230 \mathrm{~nm})$, and C $(280 \mathrm{~nm})[16,18]$, which result from single photon transitions to three lowest electronic states. All three bands are associated with the $\pi^{*} \leftarrow \pi$ transitions and there is general agreement that the $\mathrm{C}$ band is an effect of transition analogous to the ${ }^{1} \mathrm{~B}_{2 u} \leftarrow{ }^{1} \mathrm{~A}_{1 \mathrm{~g}}$ transition in benzene. Table 1 presents the calculated excitation energies for benzoic acid dimer for the first three allowed excited states obtained by the CIS and CIS(D) methods, which are compared with the experimental data. The comparison was made on the basis of the analysis of orbitals involved in electronic excitations, calculated oscillator strengths, and symmetry of the states.

Figure 2 presents the geometry of benzoic acid dimer in excited electronic state optimized at the CIS/6-311++G(d,p) and numbering the atoms. Calculated bond lengths and bond angles are summarized in Table 2 . The calculated values confirm the experimental predictions that electronic excitation leads to the shortening of one of the hydrogen bonds, whereas the other is lengthened. Also one can observe the asymmetry in calculated corresponding geometrical parameters within the aromatic rings.

The predicted dipole moment of benzoic acid dimer in the $S_{1}$ state is $0.55 \mathrm{D}$ with $0.54 \mathrm{D}$ component along axis of the dimer and $0.12 \mathrm{D}$ component perpendicular to the axis of the dimer. The calculated rotational constants for the electronically excited dimer are $\mathrm{A}=1949.2 \mathrm{MHz}, \mathrm{B}=$ 124.6 MHz, and $\mathrm{C}=117.1 \mathrm{MHz}$. 


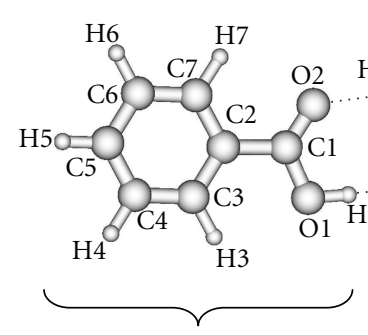

(a)

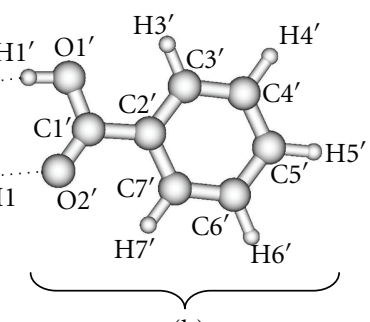

(b)

FIGURE 2: Geometry of the benzoic acid dimer in $S_{1}$ state, optimized at the CIS/6-311++G(d,p) level with numbering of atoms and labelling of the monomer units ( $\mathrm{a}$ and $\mathrm{b}$ ).

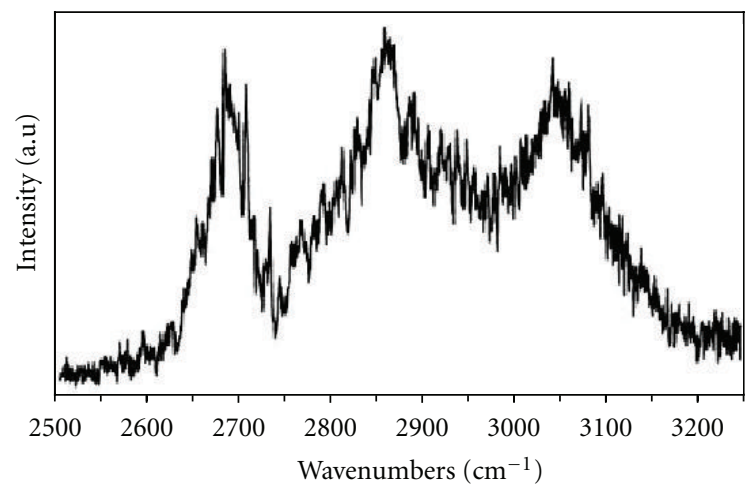

(a)

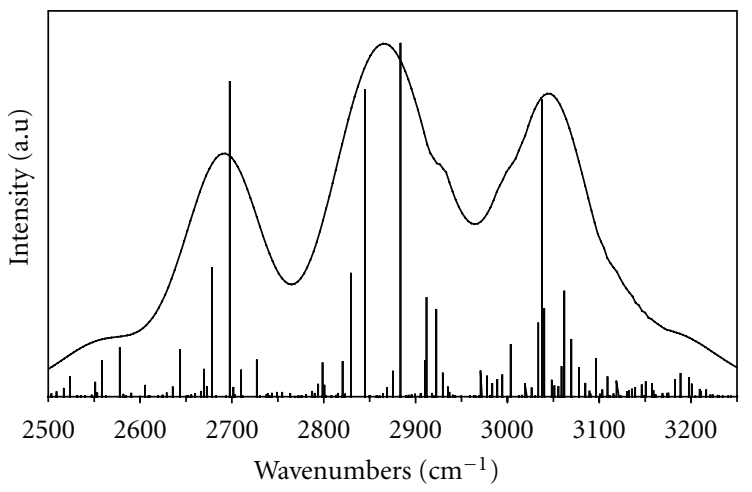

(b)

FIGURE 3: Comparison between (a) the experimental [23] and (b) theoretical (Dirac $\delta$ functions and solid line) O-H stretching IR absorption bands for benzoic acid dimer in the $S_{1}$ state.

In Table 3 we present the calculated vibrational frequencies of benzoic acid dimer in the $S_{1}$ state. This table contains also symmetry and description of the normal modes. All frequencies have been uniformly scaled by a factor of 0.9 as recommended to compensate for the neglect of mechanical anharmonicity and lack of electron correlation in the CIS method [68]. We used the MOLDEN program [69] to visualize the amplitudes of the normal modes.

The experimental FDIR (fluorescence-dip infrared) spectra of benzoic acid dimers in the excited state, taken from [23], are presented in Figure 3(a) for the $\mathrm{O}-\mathrm{H}$ stretch region. Both bands exhibit fine structures. The $v_{s}$ bands are composed of three main branches, which suggest presence of Fermi resonances. To reproduce the fine structure of experimental O-H stretching absorption bands of benzoic acid dimer, we used theoretical model presented in Section 2.2.1. The model describes complex interplay of three different vibrational couplings in a network of hydrogen bonds in benzoic acid dimer-an anharmonic coupling between the high-frequency $\mathrm{O}-\mathrm{H}$ stretching and the low-frequency intermolecular $\mathrm{O} \cdots \mathrm{O}$ stretching modes, resonance (Davydov) interaction between two intermolecular hydrogen bonds in a cyclic system, and Fermi resonance between the O-H stretching and the overtone of the $\mathrm{O}-\mathrm{H}$ in-plane bending vibrations. For the dimers in the $S_{1}$ state lowering of their symmetry upon electronic excitation was taken into account.

The experimental frequencies of the $\mathrm{O} \cdots \mathrm{O}$ hydrogen bonds stretching modes, observed in the jet-dispersed fluorescence and laser induced fluorescence spectra of benzoic acid, have been reported to be $118 \mathrm{~cm}^{-1}$ for both the $S_{0}$ and $S_{1}$ states of the dimer $[18,22]$. This frequency was taken into account in our model calculations.

To calculate energies and intensities of transitions between the ground and first excited vibrational states of the $\mathrm{O}-\mathrm{H}$ stretching vibrations, we solved the Schrödinger equations for both states. In the ground state the energies and eigenfunctions are the solutions of equations for harmonic oscillator. In the excited state they are solutions of the Schrödinger equation with the Hamiltonian (11) for $S_{1}$. The energies and eigenfunctions in the excited vibrational $\mathrm{O}_{-}$ $\mathrm{H}$ state were calculated variationally by approximating two components of the spinor (10) in the ground electronic state or four components of the spinor (17) in the excited electronic state by finite linear combinations of fourfold products of harmonic oscillator wavefunctions. We assumed the temperature $10 \mathrm{~K}$ as close to typical temperature of cold environment of free-jet expansion.

We have fitted the calculated spectra to the position of the peak with maximum intensity. In calculations of the $S_{1} I R$ spectra we took the frequencies 3367 and $3473 \mathrm{~cm}^{-1}$ from $a b$ initio CIS calculations for two O-H groups forming hydrogen bonds. We also assumed exact Fermi resonances $\left(r_{1}=r_{1}^{\prime}\right.$, $\left.r_{2}=r_{2}^{\prime}\right)$.

In order to determine optimum parameters we performed series of calculations of the $v_{s}$ stretching bands to minimize the square root deviation between experimental and 
TABLE 2: Optimized geometry of the $S_{1}$ excited-state benzoic acid dimer by the CIS/6-311++G(d,p) method.

\begin{tabular}{|c|c|c|c|}
\hline Bond lengths $(\AA)$ & & Angles (deg) & \\
\hline $\mathrm{O} 1 \cdots \mathrm{O}^{\prime}$ & 2.739 & $\mathrm{O} 1-\mathrm{H} 1 \cdots \mathrm{O}_{2}^{\prime}$ & 176.7 \\
\hline $\mathrm{O} 2 \cdots \mathrm{O}^{\prime}$ & 2.800 & $\mathrm{O}^{\prime}-\mathrm{H}^{\prime}{ }^{\prime} \cdots \mathrm{O} 2$ & 175.3 \\
\hline $\mathrm{O} 1-\mathrm{H} 1$ & 0.963 & C1-O1-H1 & 110.8 \\
\hline $\mathrm{O} 1^{\prime}-\mathrm{H} 1^{\prime}$ & 0.958 & $\mathrm{Cl}^{\prime}-\mathrm{Ol}^{\prime}-\mathrm{H1}^{\prime}$ & 110.5 \\
\hline $\mathrm{O} 2^{\prime} \cdots \mathrm{H} 1$ & 1.776 & $\mathrm{C} 1-\mathrm{O} 2 \cdots \mathrm{H}^{\prime}$ & 129.8 \\
\hline $\mathrm{O} 2 \cdots \mathrm{H}^{\prime}$ & 1.844 & $\mathrm{Cl}^{\prime}-\mathrm{O} 2^{\prime} \cdots \mathrm{H} 1$ & 131.1 \\
\hline $\mathrm{C} 1-\mathrm{O} 1$ & 1.304 & $\mathrm{O} 1-\mathrm{C} 1-\mathrm{O} 2$ & 123.2 \\
\hline $\mathrm{Cl}^{\prime}-\mathrm{O} 1^{\prime}$ & 1.315 & $\mathrm{O}^{\prime}-\mathrm{Cl}^{\prime}-\mathrm{O} 2^{\prime}$ & 122.6 \\
\hline $\mathrm{C} 1=\mathrm{O} 2$ & 1.200 & $\mathrm{O} 1-\mathrm{C} 1-\mathrm{C} 2$ & 114.2 \\
\hline $\mathrm{Cl}^{\prime}=\mathrm{O} 2^{\prime}$ & 1.213 & $\mathrm{O}^{\prime}-\mathrm{Cl}^{\prime}-\mathrm{C} 2^{\prime}$ & 114.6 \\
\hline $\mathrm{C} 1-\mathrm{C} 2$ & 1.489 & $\mathrm{O} 2-\mathrm{C} 1-\mathrm{C} 2$ & 122.6 \\
\hline $\mathrm{C} 1^{\prime}-\mathrm{C} 2^{\prime}$ & 1.447 & $\mathrm{O} 2^{\prime}-\mathrm{Cl}^{\prime}-\mathrm{C} 2^{\prime}$ & 122.8 \\
\hline C2-C3 & 1.389 & $\mathrm{C} 1-\mathrm{C} 2-\mathrm{C} 3$ & 121.4 \\
\hline $\mathrm{C} 2^{\prime}-\mathrm{C}^{\prime}$ & 1.402 & $\mathrm{C}^{\prime}-\mathrm{C}^{\prime}-\mathrm{C}^{\prime}$ & 123.5 \\
\hline C3-C4 & 1.384 & $\mathrm{C} 2-\mathrm{C} 3-\mathrm{C} 4$ & 119.9 \\
\hline $\mathrm{C} 3^{\prime}-\mathrm{C} 4^{\prime}$ & 1.414 & $\mathrm{C} 2^{\prime}-\mathrm{C} 3^{\prime}-\mathrm{C} 4^{\prime}$ & 120.4 \\
\hline C4-C5 & 1.386 & C3-C4-C5 & 120.0 \\
\hline $\mathrm{C} 4^{\prime}-\mathrm{C} 5^{\prime}$ & 1.417 & $\mathrm{C} 3^{\prime}-\mathrm{C} 4^{\prime}-\mathrm{C} 5^{\prime}$ & 121.9 \\
\hline C5-C6 & 1.387 & C4-C5-C6 & 120.3 \\
\hline $\mathrm{C} 5^{\prime}-\mathrm{C} 6^{\prime}$ & 1.397 & $\mathrm{C} 4^{\prime}-\mathrm{C} 5^{\prime}-\mathrm{C} 6^{\prime}$ & 118.1 \\
\hline C6-C7 & 1.383 & C5-C6-C7 & 119.9 \\
\hline $\mathrm{C} 6^{\prime}-\mathrm{C} 7^{\prime}$ & 1.412 & $\mathrm{C} 5^{\prime}-\mathrm{C} 6^{\prime}-\mathrm{C} 7^{\prime}$ & 120.7 \\
\hline C7-C2 & 1.390 & C6-C7-C2 & 120.0 \\
\hline $\mathrm{C} 7^{\prime}-\mathrm{C} 2^{\prime}$ & 1.436 & $\mathrm{C} 6^{\prime}-\mathrm{C} 7^{\prime}-\mathrm{C} 2^{\prime}$ & 121.3 \\
\hline C3-H3 & 1.073 & C7-C2-C3 & 120.0 \\
\hline $\mathrm{C} 3^{\prime}-\mathrm{H} 3^{\prime}$ & 1.072 & $\mathrm{C} 7^{\prime}-\mathrm{C}^{\prime}-\mathrm{C}^{\prime}$ & 117.7 \\
\hline C4-H4 & 1.075 & $\mathrm{C} 7-\mathrm{C} 2-\mathrm{C} 1$ & 118.6 \\
\hline $\mathrm{C} 4^{\prime}-\mathrm{H} 4^{\prime}$ & 1.074 & $\mathrm{C} 7^{\prime}-\mathrm{C} 2^{\prime}-\mathrm{Cl}^{\prime}$ & 118.8 \\
\hline C5-H5 & 1.076 & $\mathrm{C} 2-\mathrm{C} 3-\mathrm{H} 3$ & 119.7 \\
\hline $\mathrm{C} 5^{\prime}-\mathrm{H} 5^{\prime}$ & 1.072 & $\mathrm{C} 2^{\prime}-\mathrm{C}^{\prime}-\mathrm{H} 3^{\prime}$ & 120.1 \\
\hline C6-H6 & 1.075 & C3-C4-H4 & 119.9 \\
\hline $\mathrm{C} 6^{\prime}-\mathrm{H} 6^{\prime}$ & 1.074 & $\mathrm{C} 3^{\prime}-\mathrm{C} 4^{\prime}-\mathrm{H} 4^{\prime}$ & 118.7 \\
\hline $\mathrm{C} 7-\mathrm{H} 7$ & 1.073 & $\mathrm{C} 4-\mathrm{C} 5-\mathrm{H} 5$ & 119.9 \\
\hline \multirow[t]{5}{*}{$\mathrm{C} 7^{\prime}-\mathrm{H} 7^{\prime}$} & 1.073 & $\mathrm{C} 4^{\prime}-\mathrm{C}^{\prime}-\mathrm{H} 5^{\prime}$ & 120.6 \\
\hline & & C5-C6-H6 & 120.2 \\
\hline & & $\mathrm{C} 5^{\prime}-\mathrm{C}^{\prime}-\mathrm{H} 6^{\prime}$ & 120.6 \\
\hline & & $\mathrm{C} 6-\mathrm{C} 7-\mathrm{H} 7$ & 120.9 \\
\hline & & $\mathrm{C} 6^{\prime}-\mathrm{C} 7^{\prime}-\mathrm{H} 7^{\prime}$ & 120.3 \\
\hline
\end{tabular}

theoretical spectra. All parameters were determined with the accuracy of 0.01 .

Theoretical spectrum of benzoic acid in the $S_{1}$ state is shown and compared with the experimental spectrum in Figure 3. The optimized parameters are listed in Table 4. The theoretical spectra are shown as the Dirac delta functions and as bandshapes calculated with Gaussian functions of the optimal half width.

The reproduction of the experimental band is good. Presented results of model calculation correctly reproduce main features of the experimental spectrum. Discrepancies
TABLE 3: Calculated vibrational frequencies for $S_{1}$ state of benzoic acid dimer at the CIS/6-311++G(d,p) level ( $\nu$, stretching; $\delta$, inplane bending; $\gamma$, out-of-plane bending; $\tau$, torsion).

\begin{tabular}{cccc}
\hline \multicolumn{2}{c}{ No. Sym. } & Freq. $v\left(\mathrm{~cm}^{-1}\right)$ & Approximate description \\
\hline 1 & $\mathrm{~A}^{\prime \prime}$ & 16 & "Butterfly" monomers twisting \\
2 & $\mathrm{~A}^{\prime \prime}$ & 30 & Oop. monomers twisting \\
3 & $\mathrm{~A}^{\prime}$ & 45 & Ip. monomers twisting ("cogwheel”) \\
4 & $\mathrm{~A}^{\prime \prime}$ & 52 & Oop. monomers rocking \\
5 & $\mathrm{~A}^{\prime \prime}$ & 71 & $\tau(\mathrm{COOH})$ \\
6 & $\mathrm{~A}^{\prime \prime}$ & 80 & $\tau(\mathrm{COOH})$
\end{tabular}

Ip monomers rocking (H-bond shearing)

96

103

172

214

215

249

306

307

327

380

402

409

437

497

525

592

606

624

646

673

700

718

740

746

755

777

780

816

836

860

877

901

903

944

965

965

$44 \quad \mathrm{~A}^{\prime} \quad 976$

$\mathrm{H}$-Bonds stretching

$\tau(\mathrm{COOH})$

$\gamma(-\mathrm{COOH})(\mathrm{A})$

$\delta(-\mathrm{COOH})(\mathrm{B})$

$\gamma(-\mathrm{COOH})(\mathrm{B})$

$\delta(-\mathrm{COOH})(\mathrm{A})$

Oop. ring deform. (B)

Ip. ring deform. (B)

Oop. asym. rings deform.

Ip. ring deform. (A)

Ip. ring deform. (B)

Oop. ring deform. (A)

Oop. ring deform. (A)

$\delta(\mathrm{C}-\mathrm{COOH})(\mathrm{A})$

$\delta(\mathrm{C}-\mathrm{COOH})(\mathrm{B})$

$\delta(\mathrm{COOH})$ sciss. + ip. ring deform. (B)

Ip. ring deform. (A)

$\gamma(\mathrm{CH})(\mathrm{B})$

$\delta(\mathrm{COOH})$ sciss. + ip. ring deform. (A)

Oop. ring deform. (A)

$\gamma(\mathrm{COOH})+\gamma(\mathrm{CH})(\mathrm{B})$

$\gamma(\mathrm{CH})(\mathrm{A})$

$\delta(\mathrm{COOH})$ sciss. + ring breath. (B)

$\gamma(\mathrm{OH})+\gamma(\mathrm{CH})(\mathrm{B})$

$\gamma(\mathrm{OH})+\gamma(\mathrm{CH})(\mathrm{B})$

$\delta(\mathrm{COOH})$ sciss. + ring breath. (A)

$\gamma(\mathrm{C}-\mathrm{COOH})+\gamma(\mathrm{OH})+\gamma(\mathrm{CH})(\mathrm{B})$

$\gamma(\mathrm{C}-\mathrm{COOH})+\gamma(\mathrm{OH})+\gamma(\mathrm{CH})(\mathrm{A})$

$\gamma(\mathrm{CC})+\gamma(\mathrm{CH})(\mathrm{B})$

$\gamma(\mathrm{CC})+\gamma(\mathrm{CH})(\mathrm{A})$

$\gamma(\mathrm{OH})(\mathrm{A})$

$\gamma(\mathrm{OH})(\mathrm{B})$

$\nu(\mathrm{CC})_{\text {ring }}(\mathrm{B})$

Ring breath. (B)

$\nu(\mathrm{CC})_{\text {ring }}(\mathrm{B})$

$\gamma(\mathrm{CH})$

Ring breath. (A)

Ring breath. (A) 
TABle 3: Continued.

\begin{tabular}{|c|c|c|c|}
\hline No. & Sym. & Freq. $\nu\left(\mathrm{cm}^{-1}\right)$ & Approximate description \\
\hline 46 & $\mathrm{~A}^{\prime \prime}$ & 1004 & $\gamma_{\mathrm{A}}(\mathrm{CH})(\mathrm{A})$ \\
\hline 47 & $\mathrm{~A}^{\prime \prime}$ & 1009 & $\gamma_{\mathrm{A}}(\mathrm{CH})(\mathrm{A})$ \\
\hline 48 & $\mathrm{~A}^{\prime}$ & 1021 & $v(\mathrm{CC})_{\text {ring }}(\mathrm{B})$ \\
\hline 49 & $\mathrm{~A}^{\prime}$ & 1050 & $\nu(\mathrm{CC})_{\text {ring }}(\mathrm{A})$ \\
\hline 50 & $\mathrm{~A}^{\prime}$ & 1089 & $\nu(\mathrm{CC})_{\text {ring }}+\delta(\mathrm{CH})(\mathrm{A})$ \\
\hline 51 & $\mathrm{~A}^{\prime}$ & 1099 & $\delta(\mathrm{CC})_{\text {ring }}+\delta(\mathrm{CH})(\mathrm{B})$ \\
\hline 52 & $\mathrm{~A}^{\prime}$ & 1111 & $\nu(\mathrm{CC})_{\text {ring }}+\delta(\mathrm{CH})(\mathrm{A})$ \\
\hline 53 & $\mathrm{~A}^{\prime}$ & 1133 & $\delta(\mathrm{CH})(\mathrm{B})$ \\
\hline 54 & $\mathrm{~A}^{\prime}$ & 1155 & $\delta(\mathrm{CH})(\mathrm{A})$ \\
\hline 55 & $\mathrm{~A}^{\prime}$ & 1201 & $\nu(\mathrm{CC})_{\text {ring }}+\delta(\mathrm{CH})(\mathrm{A})$ \\
\hline 56 & $\mathrm{~A}^{\prime}$ & 1205 & $\nu(\mathrm{CC})_{\text {ring }}+\delta(\mathrm{CH})(\mathrm{B})$ \\
\hline 57 & $\mathrm{~A}^{\prime}$ & 1254 & $\delta(\mathrm{OH})(\mathrm{B})$ \\
\hline 58 & $\mathrm{~A}^{\prime}$ & 1282 & $\delta(\mathrm{OH})(\mathrm{A})$ \\
\hline 59 & $\mathrm{~A}^{\prime}$ & 1309 & $\delta(\mathrm{CH})(\mathrm{B})$ \\
\hline 60 & $\mathrm{~A}^{\prime}$ & 1312 & $\delta(\mathrm{CH})(\mathrm{A})$ \\
\hline 61 & $\mathrm{~A}^{\prime}$ & 1385 & $\delta(\mathrm{CH})(\mathrm{B})$ \\
\hline 62 & $\mathrm{~A}^{\prime}$ & 1392 & $\delta(\mathrm{CH})(\mathrm{A}+\mathrm{B})$ \\
\hline 63 & $\mathrm{~A}^{\prime}$ & 1417 & $\delta(\mathrm{CH})(\mathrm{A}+\mathrm{B})$ \\
\hline 64 & $\mathrm{~A}^{\prime}$ & 1437 & $\nu(\mathrm{CC})_{\text {ring }}+\delta(\mathrm{CH})(\mathrm{A})$ \\
\hline 65 & $\mathrm{~A}^{\prime}$ & 1445 & $\nu(\mathrm{CC})_{\text {ring }}+\delta(\mathrm{CH})(\mathrm{B})$ \\
\hline 66 & $\mathrm{~A}^{\prime}$ & 1485 & $\nu(\mathrm{CC})_{\text {ring }}+\delta(\mathrm{CH})(\mathrm{A})$ \\
\hline 67 & $\mathrm{~A}^{\prime}$ & 1533 & $\nu(\mathrm{CC})_{\text {ring }}+\delta(\mathrm{CH})(\mathrm{B})$ \\
\hline 68 & $\mathrm{~A}^{\prime}$ & 1586 & $\nu(\mathrm{CC})_{\text {ring }}(\mathrm{B})$ \\
\hline 69 & $\mathrm{~A}^{\prime}$ & 1587 & $\nu(\mathrm{CC})_{\text {ring }}(\mathrm{A})$ \\
\hline 70 & $\mathrm{~A}^{\prime}$ & 1609 & $v(\mathrm{CC})_{\text {ring }}(\mathrm{A})$ \\
\hline 71 & $\mathrm{~A}^{\prime}$ & 1653 & $\nu(\mathrm{C}=\mathrm{O})(\mathrm{B})$ \\
\hline 72 & $\mathrm{~A}^{\prime}$ & 1723 & $\nu(\mathrm{C}=\mathrm{O})(\mathrm{A})$ \\
\hline 73 & $\mathrm{~A}^{\prime}$ & 2990 & $\nu(\mathrm{CH})(\mathrm{A})$ \\
\hline 74 & $\mathrm{~A}^{\prime}$ & 3004 & $v(\mathrm{CH})(\mathrm{A})$ \\
\hline 75 & $\mathrm{~A}^{\prime}$ & 3013 & $v(\mathrm{CH})(\mathrm{A})$ \\
\hline 76 & $\mathrm{~A}^{\prime}$ & 3015 & $v(\mathrm{CH})(\mathrm{B})$ \\
\hline 77 & $\mathrm{~A}^{\prime}$ & 3020 & $v(\mathrm{CH})(\mathrm{B})$ \\
\hline 78 & $\mathrm{~A}^{\prime}$ & 3034 & $\nu(\mathrm{CH})(\mathrm{A})$ \\
\hline 79 & $\mathrm{~A}^{\prime}$ & 3038 & $v(\mathrm{CH})(\mathrm{A})$ \\
\hline 80 & $\mathrm{~A}^{\prime}$ & 3039 & $v(\mathrm{CH})(\mathrm{B})$ \\
\hline 81 & $\mathrm{~A}^{\prime}$ & 3047 & $v(\mathrm{CH})(\mathrm{B})$ \\
\hline 82 & $\mathrm{~A}^{\prime}$ & 3051 & $v(\mathrm{CH})(\mathrm{B})$ \\
\hline 83 & $\mathrm{~A}^{\prime}$ & 3367 & $\nu(\mathrm{OH})(\mathrm{A})$ \\
\hline 84 & $\mathrm{~A}^{\prime}$ & 3473 & $v(\mathrm{OH})(\mathrm{B})$ \\
\hline
\end{tabular}

The calculated frequencies were uniformly scaled by a factor of 0.9. (A) and (B) label the molecule of the dimer, which is necessary for proper mode description.

between theory and experiment are related to assumptions of the present model. Our model assumes that low-frequency $\mathrm{O} \cdots \mathrm{O}$ motion is harmonic and does not consider electrical anharmonicity. Further improvements of the model should improve agreement between theoretical and experimental bands.
TABLE 4: Optimized parameters (the frequencies of the $\mathrm{O}-\mathrm{H}$ stretching vibrations for $S_{1}$ state were taken at 3367 and $3473 \mathrm{~cm}^{-1}$ ).

\begin{tabular}{lc}
\hline & $\mathrm{S}_{1}$-state \\
\hline$\Omega($ exp. $)$ & $118 \mathrm{~cm}^{-1}$ \\
$b_{1}$ & 0.89 \\
$b_{2}$ & 0.82 \\
$V^{\text {res }}$ & -0.09 \\
$V_{1}^{\text {ah }}$ & 0.66 \\
$V_{2}^{\text {ah }}$ & 0.72 \\
$\delta^{2}$ (fixed) & 0.01 \\
Half-width & $60 \mathrm{~cm}^{-1}$ \\
\hline
\end{tabular}

2.4. Conclusions. We developed a theoretical model for an isolated hydrogen-bonded dimer of benzoic acid, in the excited electronic state, describing vibrational couplings between high- and low-frequency stretching modes in the hydrogen bonds, resonance interactions between two hydrogen bonds, and Fermi resonances between the fundamental $\mathrm{O}-\mathrm{H}$ stretching and the overtone of the $\mathrm{O}-\mathrm{H}$ in plane bending vibrations. This model was successfully used for reproduction of experimental spectrum in the excited electronic state of benzoic acid dimer. The experimental frequencies assigned to intermolecular $\mathrm{O} \cdots \mathrm{O}$ hydrogen bond stretching vibrations by $a b$ initio calculations were used in our model calculations. The calculated bandshapes and fine structures are in good agreement with the experimental ones. Our results show that considered mechanisms are the most important for hydrogen dynamics in hydrogen-bonded dimers. Infrared spectroscopy is the leading method for studying hydrogen bond properties. Quantitative theory of the IR spectra of hydrogen-bonded dimer, presented in this paper, allows for systematic study of the relation between the properties of the hydrogen bonds in the ground and excited electronic states, which is a problem of major scientific interest.

\section{Theoretical Simulation of Proton Tunneling in the Excited Electronic State of Tropolone}

3.1. Quantum Chemical Calculations. We performed $a b$ initio CIS calculations of the $\tilde{A}$ state of tropolone using the GAUSSIAN 03 program package [62]. The geometry was optimized and the vibrational frequencies were calculated by the $a b$ initio single-excitation configuration interaction [70] (CIS) with the 6-311++G(d,p). Optimized geometries are summarized in Table 5. Previous calculations of Wójcik et al. [59] performed at the CIS/6-31++G(d,p) gave slightly nonplanar geometry (in the 6-31G(d,p) basis it was planar). Our calculations confirm nonplanar structure. Increased basis set diminishes the $\mathrm{C}=\mathrm{O}$ bond length by $0.007 \AA$. The $\mathrm{O} \cdots \mathrm{O}$ distance becomes larger by $0.002 \AA$ and the $\mathrm{O}-\mathrm{H}$ distance is shorter by $0.002 \AA$.

The calculated frequencies of the normal modes of the tropolone molecule are summarized in Table 6 . The modes used in model calculations are pictured in Figure 5. All 
TABLE 5: Optimized geometries of the tropolone molecule in the Ã state by the CIS method.

\begin{tabular}{|c|c|c|c|c|c|c|c|}
\hline $\begin{array}{l}\text { Bond } \\
\text { lengths }\end{array}$ & $\begin{array}{c}\mathrm{CIS} / 6- \\
31++\mathrm{G}(\mathrm{d}, \mathrm{p}) \\
{[59](\AA)}\end{array}$ & $\begin{array}{c}\mathrm{CIS} / 6-311++\mathrm{G}(\mathrm{d}, \mathrm{p}) \\
(\AA)\end{array}$ & Bond angles & $\begin{array}{c}\text { CIS/6- } \\
31++\mathrm{G}(\mathrm{d}, \mathrm{p}) \\
{[59]\left(^{\circ}\right)}\end{array}$ & $\begin{array}{c}\mathrm{CIS} / 6- \\
311++\mathrm{G}(\mathrm{d}, \mathrm{p}) \\
\left(^{\circ}\right)\end{array}$ & Dihedral angles & $\begin{array}{c}\mathrm{CIS} / 6- \\
311++\mathrm{G}(\mathrm{d}, \mathrm{p}) \\
\left(^{\circ}\right)\end{array}$ \\
\hline $\mathrm{O} 1 \cdots \mathrm{O} 2$ & 2.534 & 2.536 & $\mathrm{O} 1-\mathrm{H} 1 \cdots \mathrm{O} 2$ & - & 115.6 & C1-C2-C3-C4 & -2.2 \\
\hline $\mathrm{O} 1-\mathrm{H} 1$ & 0.954 & 0.952 & $\mathrm{C} 1-\mathrm{O} 1-\mathrm{H} 1$ & 108.4 & 108.3 & C2-C3-C4-C5 & 8.8 \\
\hline $\mathrm{C} 2=\mathrm{O} 2$ & 1.218 & 1.211 & $\mathrm{O} 2-\mathrm{O} 1-\mathrm{H} 1$ & 44.5 & 44.5 & C3-C4-C5-C6 & -0.1 \\
\hline $\mathrm{C} 1-\mathrm{O} 1$ & 1.320 & 1.320 & $\mathrm{C} 1-\mathrm{C} 2-\mathrm{O} 2$ & 113.3 & 113.5 & C4-C5-C6-C7 & -10.0 \\
\hline $\mathrm{C} 1-\mathrm{C} 2$ & 1.512 & 1.510 & $\mathrm{C} 1-\mathrm{C} 2-\mathrm{C} 3$ & 125.3 & 125.3 & C5-C6-C7-C1 & 4.6 \\
\hline $\mathrm{C} 2-\mathrm{C} 3$ & 1.432 & 1.435 & $\mathrm{C} 2-\mathrm{C} 3-\mathrm{C} 4$ & 128.3 & 128.5 & C6-C7-C1-C2 & 8.6 \\
\hline C3-C4 & 1.398 & 1.395 & C3-C4-C5 & 129.7 & 129.9 & C7-C1-C2-C3 & -9.8 \\
\hline C4-C5 & 1.387 & 1.386 & C4-C5-C6 & 129.4 & 129.4 & $\mathrm{O} 1-\mathrm{C} 1-\mathrm{C} 2-\mathrm{O} 2$ & -5.9 \\
\hline C5-C6 & 1.425 & 1.424 & C5-C6-C7 & 128.3 & 128.4 & & \\
\hline C6-C7 & 1.387 & 1.385 & $\mathrm{C} 6-\mathrm{C} 7-\mathrm{C} 1$ & 128.8 & 129.0 & & \\
\hline $\mathrm{C} 7-\mathrm{C} 1$ & 1.388 & 1.388 & $\mathrm{C} 7-\mathrm{C} 1-\mathrm{C} 2$ & 128.7 & 128.8 & & \\
\hline C3-H3 & 1.074 & 1.074 & $\mathrm{C} 2-\mathrm{C} 3-\mathrm{H} 3$ & - & 113.0 & & \\
\hline $\mathrm{C} 4-\mathrm{H} 4$ & 1.076 & 1.076 & C3-C4-H4 & - & 115.0 & & \\
\hline C5-H5 & 1.076 & 1.076 & C4-C5-H5 & - & 115.7 & & \\
\hline C6-H6 & 1.075 & 1.074 & C5-C6-H6 & - & 115.7 & & \\
\hline $\mathrm{C} 7-\mathrm{H} 7$ & 1.076 & 1.076 & C6-C7-H7 & - & 117.0 & & \\
\hline
\end{tabular}

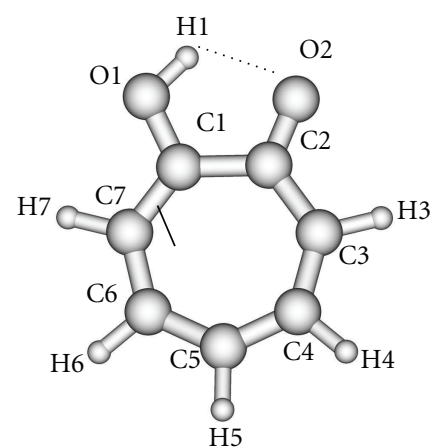

Figure 4: Geometry of the tropolone in the Ã state optimized by the CIS/6-311++G(d,p), with numbering of atoms.

frequencies have been scaled by a factor of 0.9 as recommended to compensate for the neglect of electron correlation [68]. The lowest-frequency $v_{39}$ mode strikingly changes its frequency from $109 \mathrm{~cm}^{-1}$ in the ground $\tilde{\mathrm{X}}$ state to $39 \mathrm{~cm}^{-1}$ in the excited $\tilde{\mathrm{A}}$ state [54]. These experimental frequencies are reproduced by calculations of Takada and Nakamura [58] and the present one $\left(105 \mathrm{~cm}^{-1}\right.$ and $29 \mathrm{~cm}^{-1}$, resp.). This frequency is especially important for the interpretation of the long sequence of the tunneling energy splittings suppressed by the excitation of this mode $[53,54]$.

To obtain two-dimensional potential energy surfaces for the high-frequency tunneling mode and one of selected lowfrequency modes, nearly planar modes $v_{33}$ and $v_{34}$ and outof-plane modes $\nu_{38}$ and $\nu_{39}$ we performed ab initio calculations of the normal modes of tropolone in the A state in high precision format. We applied the keyword "HPModes" in GAUSSIAN in order to obtain the high precision format (to five digits) for vibrational frequency eigenvectors in the frequency output in addition to the normal three-digit output. In the next step, beginning from the optimized equilibrium geometry of the tropolone molecule in the $\tilde{A}$ state, the series of geometries were generated. We varied the amplitudes of atomic movements for a given vibrational mode, independently for each of two coupling modes, high frequency $\mathrm{O}-\mathrm{H}$ stretching tunneling mode and one of the low-frequency modes. For each geometry mass-weighed normal coordinates have been calculated for the tunneling mode and for the low-frequency mode. The amplitudes of atomic movements were varied in the range comprising structures where distances between hydrogen atom and two oxygen atoms are equal. Such points correspond to the barrier in the double well potential surface and the corresponding structures are planar. The number of generated structures for four low-frequency modes coupled with the tunneling mode varied between 620-670 including points corresponding the barrier. For each point the single point energy was calculated at the CIS/6-311++G(d,p) level in the $\tilde{A}$ state of tropolone. In this way the one half (including barrier) of double well 
TABLE 6: Computed vibrational frequencies for the tropolone molecule in the Ã state by the CIS method.

\begin{tabular}{|c|c|c|c|}
\hline No. & Sym. & $\begin{array}{c}\text { CIS/6-31++G(d,p)[59] } \\
\text { Freq. }\left(\mathrm{cm}^{-1}\right)\end{array}$ & $\begin{array}{c}\text { CIS/6-311++G(d,p) } \\
\text { Freq. }\left(\mathrm{cm}^{-1}\right)\end{array}$ \\
\hline 1 & A & 3591 & 3594 \\
\hline 2 & A & 3041 & 3021 \\
\hline 3 & A & 3035 & 3015 \\
\hline 4 & A & 3021 & 3001 \\
\hline 5 & A & 3015 & 2994 \\
\hline 6 & A & 3003 & 2983 \\
\hline 7 & A & 1615 & 1611 \\
\hline 8 & A & 1548 & 1538 \\
\hline 9 & A & 1517 & 1506 \\
\hline 10 & A & 1478 & 1470 \\
\hline 11 & A & 1431 & 1423 \\
\hline 12 & A & 1395 & 1391 \\
\hline 13 & A & 1353 & 1347 \\
\hline 14 & A & 1281 & 1273 \\
\hline 15 & A & 1231 & 1227 \\
\hline 16 & A & 1183 & 1178 \\
\hline 17 & A & 1145 & 1139 \\
\hline 18 & A & 1059 & 1033 \\
\hline 19 & A & 945 & 998 \\
\hline 20 & A & 899 & 938 \\
\hline 21 & A & 842 & 910 \\
\hline 22 & A & 899 & 894 \\
\hline 23 & A & 842 & 832 \\
\hline 24 & A & 827 & 821 \\
\hline 25 & A & 760 & 752 \\
\hline 26 & A & 703 & 701 \\
\hline 27 & A & 675 & 673 \\
\hline 28 & A & 631 & 617 \\
\hline 29 & A & 616 & 606 \\
\hline 30 & A & 575 & 564 \\
\hline 31 & A & 516 & 515 \\
\hline 32 & A & 477 & 466 \\
\hline 33 & A & 417 & 414 \\
\hline 34 & A & 350 & 348 \\
\hline 35 & A & 347 & 344 \\
\hline 36 & A & 317 & 314 \\
\hline 37 & A & 259 & 262 \\
\hline 38 & A & 132 & 130 \\
\hline 39 & A & 37 & 29 \\
\hline
\end{tabular}

potential surface was obtained for each pair of coupled modes. The second half was obtained using the symmetry of the potential.

3.2. Model Calculations. On the basis of the ab initio calculations we constructed two-dimensional model PES's for the proton tunneling mode $\nu_{1}$ coupled to low-frequency modes of tropolone which largely affect the tunneling. These are nearly planar hydrogen-bond streching modes $\nu_{33}$ and $\nu_{34}$ and the lowest-frequency out-of-plane modes $\nu_{38}$ and $\nu_{39}$. They are shown in Figure 5 and their calculated and experimental vibrational frequencies are compared in Table 7. 


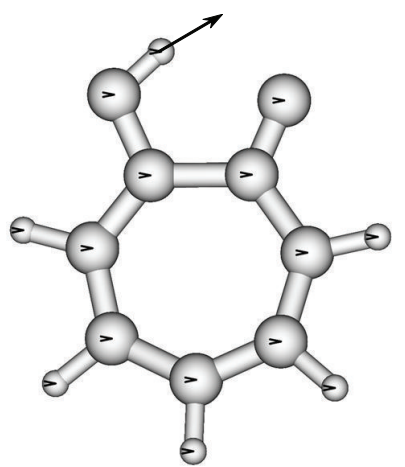

$v_{1} 3594 \mathrm{~cm}^{-1}$

(a)

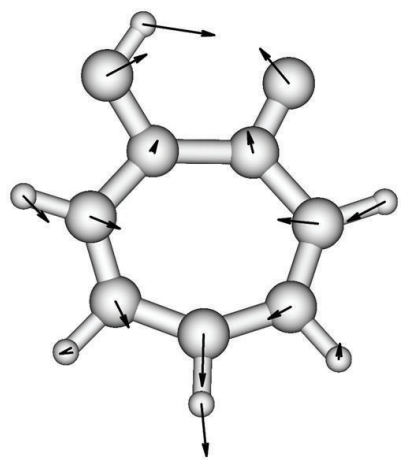

$v_{33} 414 \mathrm{~cm}^{-1}$

(b)

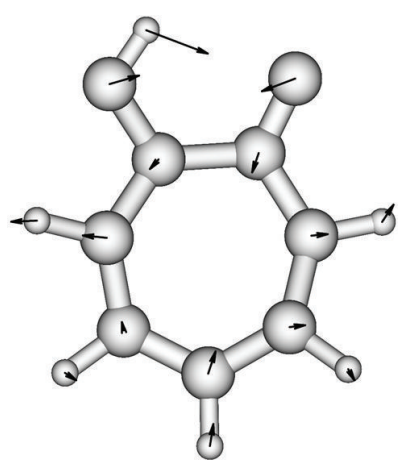

$v_{34} 348 \mathrm{~cm}^{-1}$

(c)

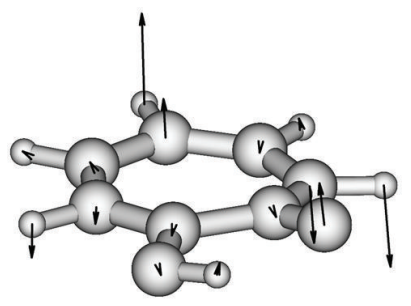

$v_{38} 130 \mathrm{~cm}^{-1}$

(d)

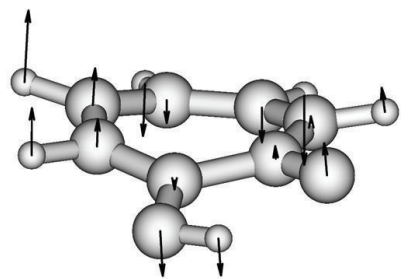

$v_{39} 29 \mathrm{~cm}^{-1}$

(e)

FIGURE 5: Selected modes of tropolone molecule in the A state calculated by the CIS/6-311++G(d,p) method.

TABLE 7: Vibrational fundamentals in the Ã state of tropolone.

\begin{tabular}{lcc}
\hline Mode & $\begin{array}{c}\text { CIS/6-311++G(d,p) } \\
\left(\mathrm{cm}^{-1}\right)\end{array}$ & $\begin{array}{c}\text { Exp. [54] } \\
\left(\mathrm{cm}^{-1}\right)\end{array}$ \\
\hline 33 & 414 & 414 \\
34 & 348 & 296 \\
38 & 130 & 171 \\
39 & 29 & 39 \\
\hline
\end{tabular}

The two-dimensional model potentials used to simulate vibrational couplings are [58]

(a) the symmetric mode coupling potential (SMC) describing couplings of the proton tunneling mode $\nu_{1}$ with the nearly planar modes $\nu_{33}$ and $\nu_{34}$ :

$$
V_{\mathrm{SMC}}=\frac{1}{8 x_{0}}\left(x-x_{0}\right)^{2}\left(x+x_{0}\right)^{2}+\frac{1}{2} \frac{\omega_{y}}{\omega_{x}}\left[y+\alpha\left(x^{2}-x_{0}^{2}\right)\right]^{2}
$$

(b) the squeezed double well potential (SQZ) describing couplings of the proton tunneling mode $\nu_{1}$ with the out-of-plane modes $\nu_{38}$ and $\nu_{39}$ :

$$
\begin{aligned}
V_{\mathrm{SMC}}= & \frac{1}{8 x_{0}}\left(x-x_{0}\right)^{2}\left(x+x_{0}\right)^{2} \\
& +\frac{1}{2 x_{0}^{2}}\left[\frac{\omega_{z}}{\omega_{x}}-\frac{\gamma\left(x^{2}-x_{0}^{2}\right)}{\left(\omega_{z} / \omega_{x}\right)}\right]^{2} z^{2},
\end{aligned}
$$

where $x$ (proton tunneling) and $y$ and $z$ (low frequency modes) denote the coordinates of the modes $\omega_{x}, \omega_{y}$, and $\omega_{z}$ are the angular frequencies $2 x_{0}$, the distance between the two minima, $\alpha$ and $\gamma$ the coupling strengths. In the formulas (18) and (19), the potentials are expressed in the units of the quantum $\hbar \omega_{x}$ and the coordinates $x, y$, and $z$ are dimensionless

$$
\begin{aligned}
& x=\tilde{x} \sqrt{\frac{m_{x} \omega_{x}}{\hbar}} \\
& y=\tilde{y} \sqrt{\frac{m_{y} \omega_{y}}{\hbar}} \\
& z=\tilde{z} \sqrt{\frac{m_{z} \omega_{z}}{\hbar}} .
\end{aligned}
$$

where $\tilde{x}, \tilde{y}$, and $\tilde{z}$ denote the dimensional coordinates and $m_{x}, m_{y}$, and $m_{z}$ are the effective masses. The model potentials (18) and (19) have been fitted to the grids of 

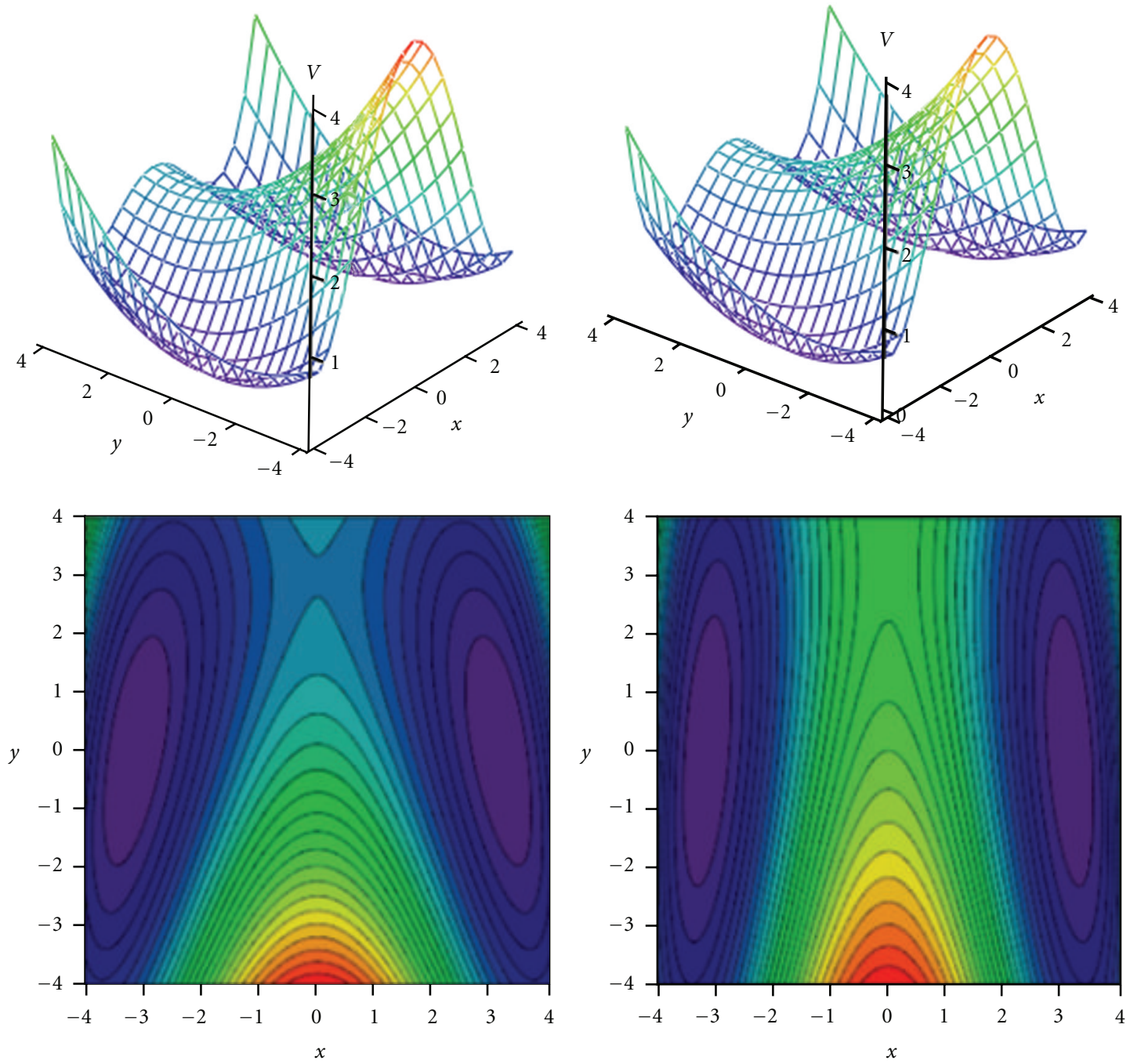

(a)

(b)

FIGURE 6: Surface plots and contour maps of the two-dimensional SMC potentials obtained from the $a b$ initio CIS calculations for: (a) coupling of $v_{1}$ and $v_{33}$ modes, (b) coupling of $v_{1}$ and $v_{34}$ modes.

energy single points obtained from the ab initio calculations for each pair: high-frequency tunneling mode and low-frequency mode. The obtained potentials are shown as a surface plots and as a contour plots in Figures 6 and 7. The energy is expressed in the units of the quantum of the highfrequency tunneling mode $v_{1}$. The optimal values of parameters $x_{0}, \alpha, \gamma$, for the model potentials have been found through the nonlinear least-squares method with the Levenberg-Marquardt algorithm [71, 72]. These parameters have been used in subsequent calculations of the energy splittings and they are listed in Table 8 . The parameter $\alpha$ describing coupling between the $\mathrm{O}-\mathrm{H}$ stretching mode $v_{1}$ and the mode $v_{33}$ or $v_{34}$ represents an analogue of a linear distortion parameter $b_{2}$ used for theoretical reproduction of the $\mathrm{O}-\mathrm{H}$ infrared bandshape of tropolone [70]. Infrared spectra of tropolone in the excited electronic state $\tilde{A}$ are yet unknown, but the value of the parameter $b_{2}=0.4$, describing the coupling between the $v_{1}$ and $v_{34}$ modes and used to reproduce IR
TABLE 8: Parameters of the two-dimensional model potentials given by (18) and (19).

\begin{tabular}{lccc}
\hline Mode & $X_{0}$ & $\alpha$ & $\gamma$ \\
\hline 33 & 3.15 & 0.301 & \\
34 & 3.15 & 0.323 & \\
38 & 3.15 & & 0.006640 \\
39 & 3.15 & & 0.000911 \\
\hline
\end{tabular}

bandshape of tropolone in the ground electronic state $\tilde{X}$ [73], is consistent with the value of the parameter $\alpha=0.323$ presently used to reproduce the dependence of the tunneling splittings on excitations of the $v_{34}$ mode. Both different spectroscopic facts have the same origin, the anharmonictype coupling in the potential energy between the two $\mathrm{O}-\mathrm{H}$ and $\mathrm{O} \cdots \mathrm{O}$ vibrations. 

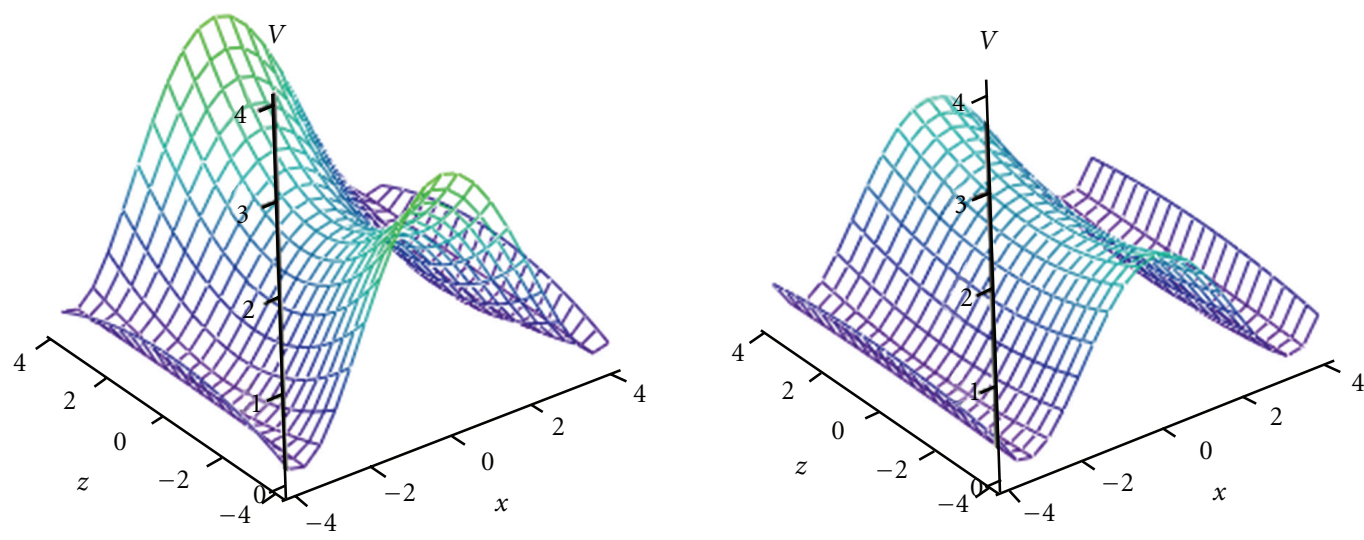

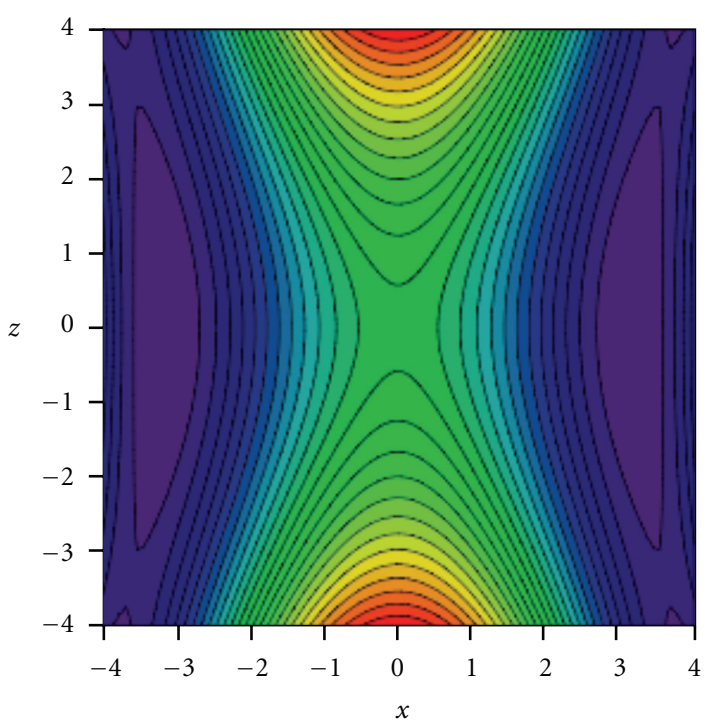

(a)

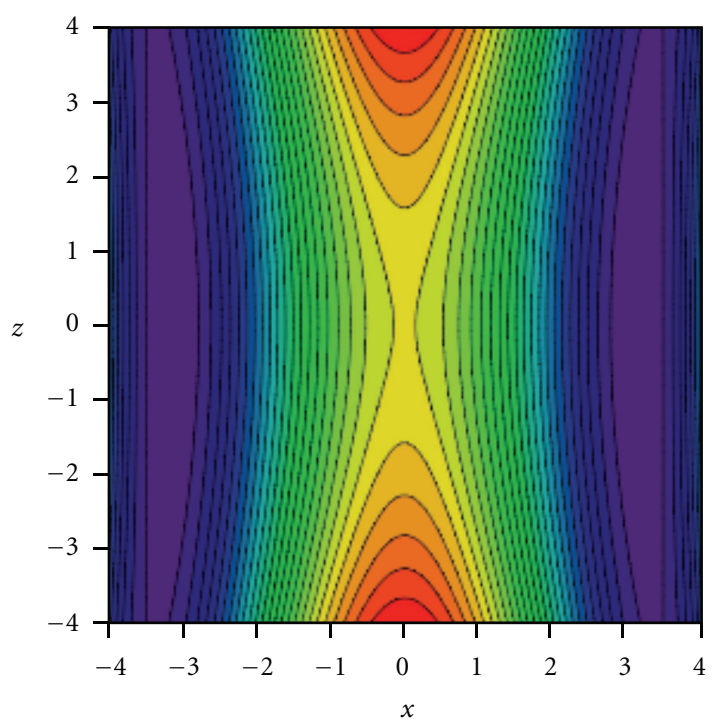

(b)

FIGURE 7: Surface plots and contour maps of the two-dimensional SQZ potentials obtained from the ab initio CIS calculations for: (a) coupling of $v_{1}$ and $v_{38}$ modes, (b) coupling of $v_{1}$ and $v_{39}$ modes.

Tunneling energy splittings have been calculated variationally by the DVR method $[74,75]$. The results are presented in Table 9. Comparison between calculated and experimental splittings shows that two-dimensional model potentials fitted to the grids of energies calculated by the CIS/6-311++G(d,p) method very well reproduce experimentally observed tunneling splittings and their dependence on vibrational excitations in tropolone. The calculated potential energy surfaces quantitatively explain increase of the tunneling splittings with excitations of the nearly planar $\nu_{33}$ and $\nu_{34}$ modes and decrease of the splittings with excitation of the out-of-plane $\nu_{38}$ and $\nu_{39}$ modes. Especially striking is long sequence of monotonic decrease of the energy splittings accompanying excitations of the out-of-plane $\nu_{39}$ mode quantitatively reproduced by our calculations. Our calculations predict monotonic increase of tunneling splitting with vibrational excitations for the nearly planar $v_{34}$ mode. The experimental results show an oscillatory behavior of the energy splitting as a function of the vibrational quantum number for this mode. Our model cannot explain such behavior. According to Takada and Nakamura [58], the energy splitting oscillates with respect to quantum number in the case of antisymmetric mode coupling potential in socalled mixed tunneling region which can be an explanation of the observed effect.

Present approach constitutes improvement of the previous work [59] which used the same model potentials, given by (18) and (19); however the method to obtain parameters was different. The coupling parameters $\alpha$ and $\gamma$ were obtained from approximate formulas taking into account only two structures in the $\tilde{A}$ state, stable structure and saddle point structure (transition state). Parameters obtained in such way were different, especially values of $\gamma$ were by one order of magnitude lower than values obtained in this work. Previous model calculations reproduced quantitatively experimental tunneling energy splitting in the vibrationally ground state of tropolone but only qualitatively changes of 
TABLE 9: Energy splittings calculated for the two-dimensional model potentials for the Ã state of tropolone.

\begin{tabular}{|c|c|c|c|}
\hline Band & CIS/6-31++G** $\left(\mathrm{cm}^{-1}\right)[59]$ & $\mathrm{CIS} / 6-311++\mathrm{G}^{* *}\left(\mathrm{~cm}^{-1}\right)$ & Exp. $[53,54]\left(\mathrm{cm}^{-1}\right)$ \\
\hline $33^{0}$ & 22.9 & 20.3 & 20 \\
\hline $33^{1}$ & 25.1 & 30.2 & 33 \\
\hline $33^{2}$ & 27.4 & 41.2 & \\
\hline $33^{3}$ & 29.7 & 53.2 & \\
\hline $34^{0}$ & 20.7 & 20.0 & 20 \\
\hline $34^{1}$ & 24.7 & 28.0 & 31 \\
\hline $34^{2}$ & 28.8 & 36.7 & 29 \\
\hline $34^{3}$ & 33.2 & 46.1 & \\
\hline $38^{0}$ & 24.5 & 24.4 & 20 \\
\hline $38^{1}$ & 24.0 & 18.2 & \\
\hline $38^{2}$ & 23.5 & 9.4 & 5 \\
\hline $38^{3}$ & 23.0 & 5.5 & \\
\hline $39^{0}$ & 24.7 & 22.9 & 20 \\
\hline $39^{1}$ & 24.5 & 20.1 & \\
\hline $39^{2}$ & 24.3 & 16.8 & 8 \\
\hline $39^{3}$ & 24.1 & 12.9 & \\
\hline $39^{4}$ & 23.9 & 10.5 & 6 \\
\hline $39^{5}$ & 23.7 & 7.9 & \\
\hline $39^{6}$ & 23.5 & 5.3 & 5 \\
\hline $39^{7}$ & 23.3 & 3.5 & \\
\hline $39^{8}$ & 23.1 & 1.6 & 2 \\
\hline
\end{tabular}

the tunneling splittings with excitations of low-frequency modes.

Previously there have been other attempts to interpret tunneling splittings in the $\tilde{\mathrm{A}}$ state of tropolone, by Vener et al. [56] and Smedarchina et al. [57] Vener et al. used an adiabatic description in a model three-dimensional potential based on the ab initio CIS/6-31G calculations. Their approach was not successful in describing the dynamics of the excited state. Smedarchina et al. employed an instanton method combined with the PES obtained by the $a b$ initio CIS/6-31G** calculations. They were able to obtain satisfactory agreement between theory and experiment for linearly coupled modes, however they had to adjust the adiabatic barrier height. Our present results do not require such adjustment and present pure quantum mechanical approach to the problem of tunneling splittings in the excited state of tropolone. Burns et al. have also provided detailed quantum mechanical computations for the vibrations and potential energy surface properties of tropolone in its lowest pi*-pi electronic excited state [76].

In this approach we do not deal with the other low-frequency modes. The modes we took are typical ones to explain the effects of vibrational excitation on tunneling. The other modes are either higher-frequency modes or not hydrogen-bond stretching modes (e.g., $v_{35}, v_{36}, v_{37}$ ). The model potentials used in this paper are not adequate to describe the influence of these modes on proton tunneling.
3.3. Conclusions. The proton tunneling dynamics of tropolone in the excited $\tilde{A}$ state have been studied by performing the high accuracy quantum mechanical calculations of the potential energy surfaces and fitting them by two-dimensional model potentials. The tunneling energy splittings for different vibrationally excited states of low-frequency modes have been calculated and compared with the available experimental data. The experimentally observed promotion of the tunneling by the excitation of the planar $v_{33}$ and $\nu_{34}$ modes and suppression by the excitation of the out-ofplane $\nu_{38}$ and $\nu_{39}$ modes have been reproduced quantitatively by our calculations. They reproduce the long sequence of monotonic decrease of the tunneling splittings accompanying excitations of the out-of-plane $v_{39}$ mode.

\section{Acknowledgments}

Figures 1-3 and Tables 1-4 are reprinted with permission from M. Boczar, Ł. Boda and M.J. Wójcik, J. Chem. Phys. 127, 084307 (2007). Copyright 2007, American Institute of Physics. Figures 4-7 and Tables 5-9 are reprinted with permission from M.J. Wójcik, Ł. Boda and M. Boczar, J. Chem. Phys. 130, 164306 (2009). Copyright 2009, American Institute of Physics.

\section{References}

[1] Y. Maréchal and A. Witkowski, "Infrared spectra of H-bonded systems," The Journal of Chemical Physics, vol. 48, no. 8, pp. 
3697-3705, 1968.

[2] M. J. Wójcik, "Theory of the infrared spectra of the hydrogen bond in molecular crystals," International Journal of Quantum Chemistry, vol. 10, pp. 747-760, 1976.

[3] A. Witkowski and M. Wójcik, "Infrared spectra of hydrogen bond a general theoretical model," Chemical Physics, vol. 1, pp. 9-16, 1973.

[4] M. J. Wójcik, "Fermi resonance in dimers : a model study," Molecular Physics, vol. 36, pp. 1757-1767, 1978.

[5] O. Henri-Rousseau and D. Chamma, "IR spectral density of weak H-bonded complexes involving damped Fermi resonances. I. Quantum theory," Chemical Physics, vol. 229, no. 1, pp. 37-50, 1998.

[6] A. M. Yaremko, H. Ratajczak, J. Baran, A. J. Barnes, E. V. Mozdor, and B. Silvi, "Theory of profiles of hydrogen bond stretching vibrations: Fermi-Davydov resonances in hydrogen-bonded crystals," Chemical Physics, vol. 306, no. 13, pp. 57-70, 2004.

[7] N. Rösch and M. A. Ratner, "Model for the effects of a condensed phase on the infrared spectra of hydrogen-bonded systems," The Journal of Chemical Physics, vol. 61, no. 8, pp. 3344-3351, 1974.

[8] S. Bratos, "Profiles of hydrogen stretching ir bands of molecules with hydrogen bonds: a stochastic theory. I. Weak and medium strength hydrogen bonds," The Journal of Chemical Physics, vol. 63, no. 8, pp. 3499-3509, 1975.

[9] G. N. Robertson and J. Yarwood, "Vibrational relaxation of hydrogen-bonded species in solution. I. Theory," Chemical Physics, vol. 32, no. 2, pp. 267-282, 1978.

[10] O. Henri-Rousseau and P. Blaise, "The infrared spectral density of weak hydrogen bonds within the linear response theory," Advances in Chemical Physics, vol. 103, p. 1, 1998.

[11] P. Blaise, M. J. Wójcik, and O. Henri-Rousseau, "Theoretical interpretation of the line shape of the gaseous acetic acid cyclic dimer," The Journal of Chemical Physics, vol. 122, no. 6, Article ID 064306, pp. 1-12, 2005.

[12] M. Ito, H. Tsukioka, and S. Imanishi, "Effect of temperature on ultraviolet absorption spectra of benzoic acids and its relation to hydrogen bonding," Journal of the American Chemical Society, vol. 82, no. 7, pp. 1559-1564, 1960.

[13] H. Baba and M. Kitamura, "Molecular association and emission spectra of benzoic acid," Journal of Molecular Spectroscopy, vol. 41, no. 2, pp. 302-309, 1972.

[14] J. C. Baum and D. S. McClure, "The ultraviolet transitions of benzoic acid. 4. High-resolution spectral studies of hydrogen bonding in the excited states of the benzoic acid dimer," Journal of the American Chemical Society, vol. 102, no. 2, pp. 720-727, 1980.

[15] J. C. Baum and D. S. McClure, "The ultraviolet transitions of benzoic acid. 2. Hydrogen bonding in the ground and excited states," Journal of the American Chemical Society, vol. 101, no. 9, pp. 2340-2343, 1979.

[16] J. C. Baum and D. S. McClure, "The ultraviolet transitions of benzoic acid. 1. Interpretation of the singlet absorption spectrum," Journal of the American Chemical Society, vol. 101, no. 9, pp. 2335-2339, 1979.

[17] J. C. Baum, "The ultraviolet transitions of benzoic acid. 3. Effects of hydrogen bonding on the emission properties," Journal of the American Chemical Society, vol. 102, no. 2, pp. 716719, 1980.

[18] D. E. Poeltl and J. K. McVey, "Laser induced fluorescence excitation spectrum of jet-cooled benzoic acid dimers," The Journal of Chemical Physics, vol. 78, no. 7, pp. 4349-4355,
1983.

[19] D. E. Poeltl and J. K. McVey, "Excited-state dynamics of hydrogen-bonded dimers of benzoic acid," The Journal of Chemical Physics, vol. 80, no. 5, pp. 1801-1811, 1984.

[20] Y. Tomioka, H. Abe, N. Mikami, and M. Ito, "Electronic spectra of benzoic acid in a supersonic free jet," Journal of Physical Chemistry, vol. 88, no. 11, pp. 2263-2270, 1984.

[21] K. Remmers, W. L. Meerts, and I. Ozier, "Proton tunneling in the benzoic acid dimer studied by high resolution ultraviolet spectroscopy," The Journal of Chemical Physics, vol. 112, no. 24, pp. 10890-10894, 2000.

[22] C. K. Nandi and T. Chakraborty, "Hydrogen bond-induced vibronic mode mixing in benzoic acid dimer: a laser-induced fluorescence study," The Journal of Chemical Physics, vol. 120, no. 18, pp. 8521-8527, 2004.

[23] G. M. Florio, E. L. Sibert, and T. S. Zwier, "Fluorescence-dip IR spectra of jet-cooled benzoic acid dimer in its ground and first excited singlet states," Faraday Discussions, vol. 118, pp. 315-330, 2001.

[24] G. M. Florio, T. S. Zwier, E. M. Myshakin, K. D. Jordan, and E. L. Sibert III, "Theoretical modeling of the $\mathrm{OH}$ stretch infrared spectrum of carboxylic acid dimers based on first-principles anharmonic couplings," The Journal of Chemical Physics, vol. 118, no. 4, pp. 1735-1746, 2003.

[25] H. T. Flakus and M. Chelmecki, "Infrared spectra of the hydrogen bond in benzoic acid crystals: temperature and polarization effects," Spectrochimica Acta, vol. 58, no. 1, pp. 179-196, 2002.

[26] H. T. Flakus, "A new approach to the problem of the hydrogen bond spectra of the adipic acid crystal: the polarization and temperature effects," Journal of Molecular Structure, vol. 285, no. 3, pp. 281-292, 1993.

[27] H. R. Flakus and A. Bryk, "Strong-coupling mechanism for interpretation of the IR spectra of the hydrogen bonded imidazole crystal," Journal of Molecular Structure, vol. 372, no. 2-3, pp. 215-227, 1995.

[28] H. T. Flakus and A. Bryk, "An extended "strong-coupling" model of the IR spectral properties of molecular crystals with four H-bonds in a unit cell: the imidazole-type crystals," Journal of Molecular Structure, vol. 372, no. 2-3, pp. 229-240, 1995.

[29] H. T. Flakus and A. Miros, "Infrared spectra of the hydrogen bonded glutaric acid crystals: polarization and temperature effects," Journal of Molecular Structure, vol. 484, no. 1-3, pp. 103-115, 1999.

[30] M. Boczar, K. Szczeponek, M. J. Wójcik, and C. Paluszkiewicz, "Theoretical modeling of infrared spectra of benzoic acid and its deuterated derivative," Journal of Molecular Structure, vol. 700, no. 1-3, pp. 39-48, 2004.

[31] M. Boczar, Ł. Boda, and M. J. Wójcik, "Theoretical model of infrared spectra of hydrogen bonds in molecular crystals and its application to interpretation of infrared spectra of 1methylthymine," The Journal of Chemical Physics, vol. 125, no. 8, Article ID 084709, 2006.

[32] P. Blaise, M. J. Wójcik, and O. Henri-Rousseau, "Theoretical interpretation of the line shape of the gaseous acetic acid cyclic dimer," The Journal of Chemical Physics, vol. 122, no. 6, Article ID 064306, pp. 1-12, 2005.

[33] M. Boczar, Ł. Boda, and M. J. Wójcik, "Theoretical model for a tetrad of hydrogen bonds and its application to interpretation of infrared spectra of salicylic acid," The Journal of Chemical Physics, vol. 124, no. 8, Article ID 084306, 2006.

[34] G.-J. Zhao, J.-Y. Liu, L.-C. Zhou, and K.-L. Han, "Site-selective photoinduced electron transfer from alcoholic solvents to the 
chromophore facilitated by hydrogen bonding: a new fluorescence quenching mechanism," Journal of Physical Chemistry B, vol. 111, no. 30, pp. 8940-8945, 2007.

[35] G.-J. Zhao and K.-L. Han, "Early time hydrogen-bonding dynamics of photoexcited coumarin 102 in hydrogen-donating solvents: theoretical study," Journal of Physical Chemistry A, vol. 111, no. 13, pp. 2469-2474, 2007.

[36] G.-J. Zhao and K.-L. Han, "Site-specific solvation of the photoexcited protochlorophyllide $a$ in methanol: formation of the hydrogen-bonded intermediate state induced by hydrogenbond strengthening," Biophysical Journal, vol. 94, p. 38, 2008.

[37] G.-J. Zhao and K.-L. Han, "Effects of hydrogen bonding on tuning photochemistry: concerted hydrogen-bond strengthening and weakening," ChemPhysChem, vol. 9, no. 13, pp. 1842-1846, 2008.

[38] P.-O. Löwdin, "Proton tunneling in DNA and its biological implications," Reviews of Modern Physics, vol. 35, pp. 724-732, 1963.

[39] H. Nakamura, "Theoretical studies of chemical dynamics: overview of some fundamental mechanisms," Annual Review of Physical Chemistry, vol. 48, no. 1, pp. 299-328, 1997.

[40] V. A. Benderskii, I. S. Irgibaeva, E. V. Vetoshkin, and H. P. Trommsdorff, "Tunneling splittings in vibrational spectra of non-rigid molecules. VIII. Six-dimensional tunneling dynamics of hydrogen peroxide and its isotopomers," Chemical Physics, vol. 262, no. 2-3, pp. 369-391, 2000.

[41] V. A. Benderskii, E. V. Vetoshkin, I. S. Irgibaeva, and H. P. Trommsdorff, "Tunneling splittings in vibrational spectra of non-rigid molecules: IX. Malonaldehyde and its isotopomers as a test case for fully coupled multidimensional tunneling dynamics," Chemical Physics, vol. 262, no. 2-3, pp. 393-422, 2000.

[42] M. V. Pak and S. Hammes-Schiffer, "Electron-proton correlation for hydrogen tunneling systems," Physical Review Letters, vol. 92, no. 10, p. 103002, 2004.

[43] C. S. Tautermann, A. F. Voegele, and K. R. Liedl, "The groundstate tunneling splitting of various carboxylic acid dimers," The Journal of Chemical Physics, vol. 120, no. 2, pp. 631-637, 2004.

[44] C. S. Tautermann, M. J. Loferer, A. F. Voegele, and K. R. Liedl, "Double hydrogen tunneling revisited: the breakdown of experimental tunneling criteria," The Journal of Chemical Physics, vol. 120, no. 24, pp. 11650-11657, 2004.

[45] G. V. Mil'nikov, K. Yagi, T. Taketsugu, H. Nakamura, and K. Hirao, "Simple and accurate method to evaluate tunneling splitting in polyatomic molecules," The Journal of Chemical Physics, vol. 120, no. 11, pp. 5036-5045, 2004.

[46] K. Yagi, G. V. Mil'nikov, T. Taketsugu, K. Hirao, and H. Nakamura, "Effect of out-of-plane vibration on the hydrogen atom transfer reaction in malonaldehyde," Chemical Physics Letters, vol. 397, no. 4-6, pp. 435-440, 2004.

[47] G. V. Mil'nikov and H. Nakamura, "Instanton theory for the tunneling splitting of low vibrationally excited states," The Journal of Chemical Physics, vol. 122, no. 12, p. 124311, 2005.

[48] G. V. Mil'nikov, T. Ishida, and H. Nakamura, "Tunneling splitting of energy levels and rotational constants in the vinyl radical $\mathrm{C}_{2} \mathrm{H}_{3}$," Journal of Physical Chemistry A, vol. 110, no. 16, pp. 5430-5435, 2006.

[49] G. V. Mil'nikov and H. Nakamura, "Tunneling splitting and decay of metastable states in polyatomic molecules: invariant instanton theory," Physical Chemistry Chemical Physics, vol. 10, pp. 1374-1393, 2008.

[50] A. C. P. Alves and J. M. Hollas, "The near ultra-violet absorption spectrum of tropolone vapour," Molecular Physics, vol. 25, pp. 1305-1314, 1973.

[51] R. L. Redington and T. E. Redington, "Tropolone monomer. Vibrational spectrum and proton tunneling," Journal of Molecular Spectroscopy, vol. 78, no. 2, pp. 229-247, 1979.

[52] Y. Tomioka, M. Ito, and N. Mikami, "Electronic spectra of tropolone in a supersonic free jet. Proton tunneling in the $S_{1}$ state," Journal of Physical Chemistry, vol. 87, no. 22, pp. 44014405, 1983.

[53] R. L. Redington, Y. Chen, G. J. Scherer, and R. W. Field, "Laser fluorescence excitation spectrum of jet-cooled tropolone: the $\tilde{\mathrm{A}}^{1} \mathrm{~B}_{2}-\mathrm{X} \sim{ }^{1} \mathrm{~A}_{1}$ system," The Journal of Chemical Physics, vol. 88, no. 2, pp. 627-633, 1988.

[54] H. Sekiya, Y. Nagashima, and Y. Nishimura, "Electronic spectra of jet-cooled tropolone. Effect of the vibrational excitation on the proton tunneling dynamics," The Journal of Chemical Physics, vol. 92, no. 10, pp. 5761-5769, 1990.

[55] K. Nishi, H. Sekiya, H. Kawakami, A. Mori, and Y. Nishimura, "Coupling of internal rotation of methyl group with proton transfer in the $\mathrm{S}_{1}$ state of 5-methyltropolone," The Journal of Chemical Physics, vol. 109, no. 5, pp. 1589-1592, 1998.

[56] M. V. Vener, S. Scheiner, and N. D. Sokolov, "Theoretical study of hydrogen bonding and proton transfer in the ground and lowest excited singlet states of tropolone," The Journal of Chemical Physics, vol. 101, no. 11, pp. 9755-9765, 1994.

[57] Z. Smedarchina, W. Siebrand, and M. Z. Zgierski, "Modespecific hydrogen tunneling in tropolone: an instanton approach," The Journal of Chemical Physics, vol. 104, no. 4, pp. 1203-1212, 1996.

[58] S. Takada and H. Nakamura, "Effects of vibrational excitation on multidimensional tunneling: general study and proton tunneling in tropolone," The Journal of Chemical Physics, vol. 102, no. 10, pp. 3977-3992, 1995.

[59] M. J. Wójcik, H. Nakamura, S. Iwata, and W. Tatara, "Theoretical study of multidimensional proton tunneling in the excited state of tropolone," The Journal of Chemical Physics, vol. 112, no. 14, pp. 6322-6328, 2000.

[60] M. Boczar, Ł. Boda, and M. J. Wójcik, "Theoretical modeling of the $\mathrm{O}-\mathrm{H}$ stretching IR bands of hydrogen-bonded dimers of benzoic acid in $\mathrm{S}_{0}$ and $\mathrm{S}_{1}$ electronic states," The Journal of Chemical Physics, vol. 127, no. 8, Article ID 084307, 2007.

[61] M. J. Wójcik, Ł. Boda, and M. Boczar, "Theoretical study of proton tunneling in the excited state of tropolone," The Journal of Chemical Physics, vol. 130, no. 16, Article ID 164306, 2009.

[62] M. J. Frisch, G. W. Trucks, H. B. Schlegel et al., GAUSSIAN 03, Revision D.01, Gaussian, Wallingford, Conn, USA, 2004.

[63] J. B. Foresman, M. Head-Gordon, J. A. Pople, and M. J. Frisch, "Toward a systematic molecular orbital theory for excited states," Journal of Physical Chemistry, vol. 96, no. 1, pp. 135149, 1992.

[64] M. Head-Gordon, R. J. Rico, M. Oumi, and T. J. Lee, "A doubles correction to electronic excited states from configuration interaction in the space of single substitutions," Chemical Physics Letters, vol. 219, pp. 21-29, 1994.

[65] M. Head-Gordon, D. Maurice, and M. Oumi, "A perturbative correction to restricted open shell configuration interaction with single substitutions for excited states of radicals," Chemical Physics Letters, vol. 246, no. 1-2, pp. 114-121, 1995.

[66] C. H. Longuet-Higgins, in Advances in Spectroscopy, H. W. Thompson, Ed., vol. 2, p. 429, Wiley-Interscience, New York, NY, USA, 1961.

[67] R. L. Fulton and M. Gouterman, "Vibronic coupling. I. Mathematical treatment for two electronic states," The Journal of Chemical Physics, vol. 35, no. 3, pp. 1059-1071, 1961.

[68] W. J. Hehre, L. Radom, P. V. R. Schleyer, and J. A. Pople, Ab 
Initio Molecular Orbital Theory, Wiley, New York, NY, USA, 1986.

[69] G. Schaftenaar, "MOLDEN: a portable electron density program," QCPE Bulletin, vol. 619, p. 12, 1992.

[70] J. B. Foresman, M. Head-Gordon, J. A. Pople, and M. J. Frisch, "Toward a systematic molecular orbital theory for excited states," Journal of Physical Chemistry, vol. 96, no. 1, pp. 135$149,1992$.

[71] K. Levenberg, "A method for the solution of certain problems in least squares," Quarterly of Applied Mathematics, vol. 2, pp. 164-168, 1944.

[72] D. Marquardt, "An algorithm for least-squares estimation of nonlinear parameters," Journal on Applied Mathematics, vol. 11, pp. 431-441, 1963.

[73] M. J. Wójcik, M. Boczar, and M. Stoma, "Spectroscopic and theoretical study of vibrational spectra of hydrogen-bonded tropolone," International Journal of Quantum Chemistry, vol. 73, no. 3, pp. 275-282, 1999.

[74] J. C. Light, I. P. Hamilton, and J. V. Lill, "Generalized discrete variable approximation in quantum mechanics ${ }^{a)}$," The Journal of Chemical Physics, vol. 82, pp. 1400-1409, 1985.

[75] M. Whitnell and J. C. Light, "Efficient pointwise representations for vibrational wave functions: eigenfunctions of H3(+)," The Journal of Chemical Physics, vol. 90, p. 1774, 1989.

[76] L. A. Burns, D. Murdock, and P. H. Vaccaro, "An exploration of electronic structure and nuclear dynamics in tropolone: II. the $\tilde{\mathrm{A}}^{1} \mathrm{~B}_{2}(\pi \pi)$ excited state," The Journal of Chemical Physics, vol. 130, no. 14, Article ID 144304, 2009. 

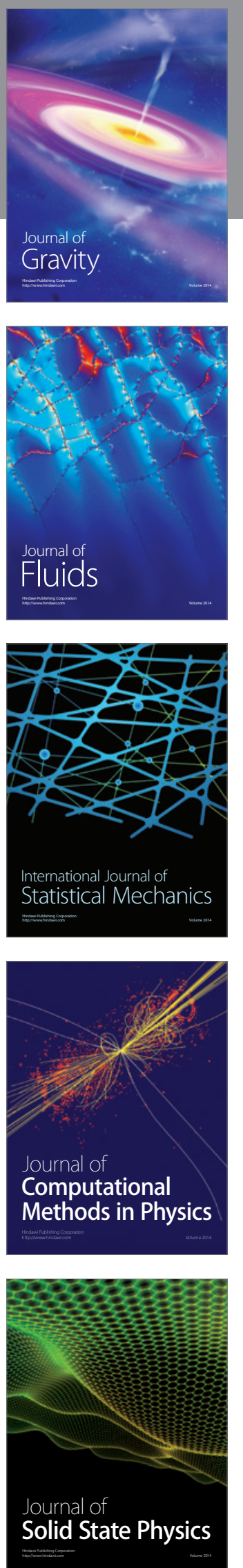

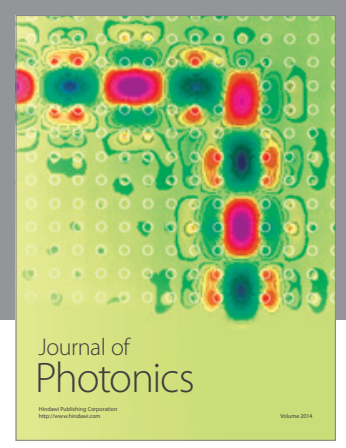

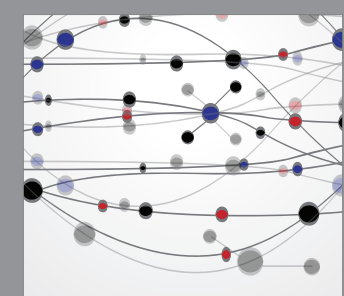

The Scientific World Journal
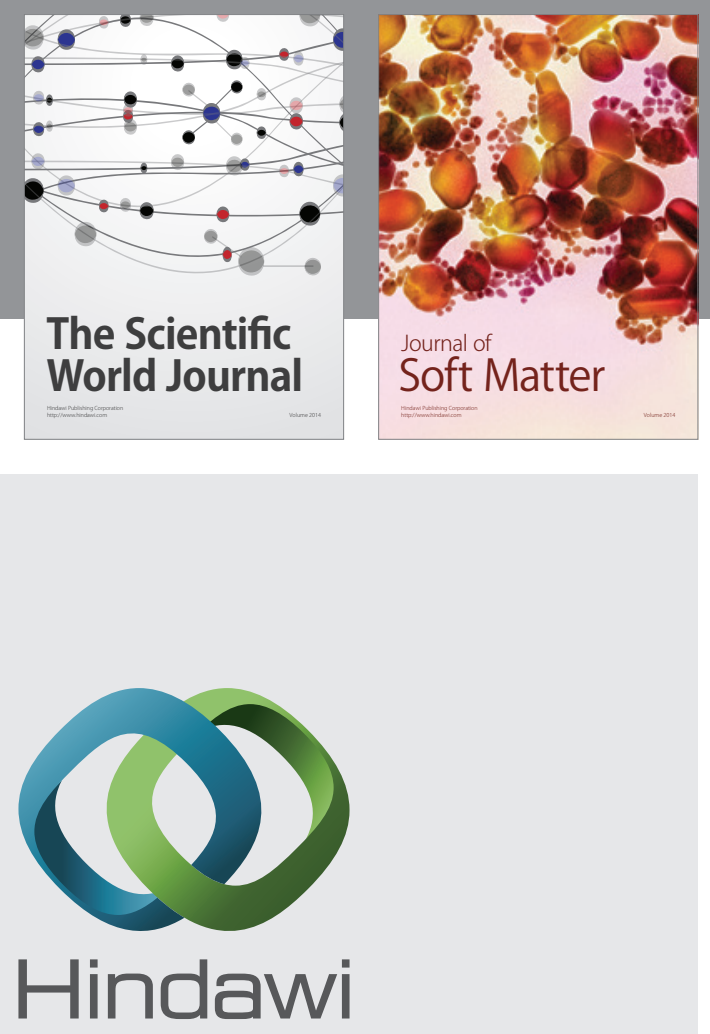

Submit your manuscripts at

http://www.hindawi.com
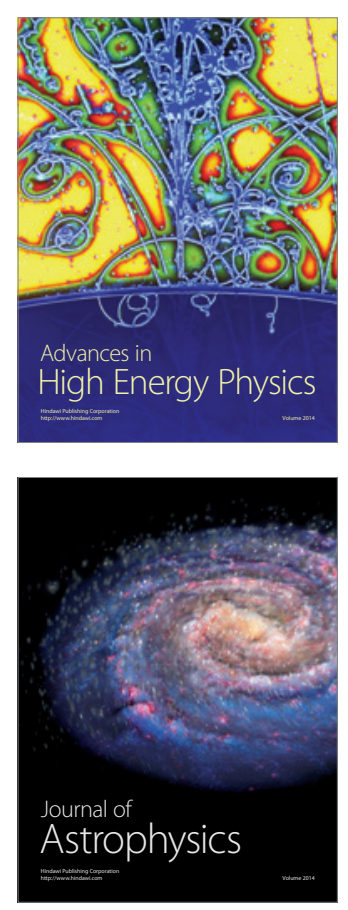
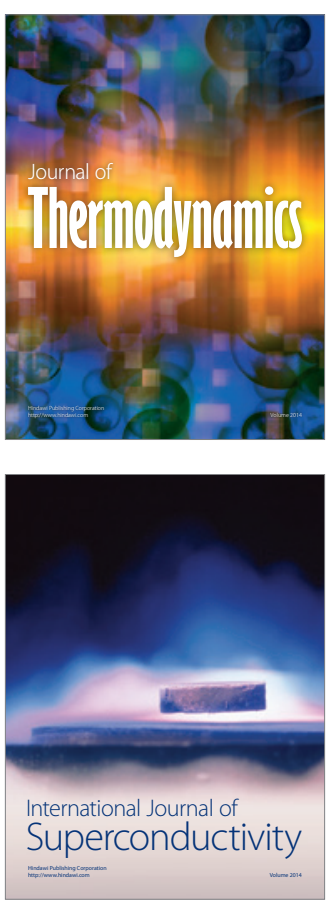
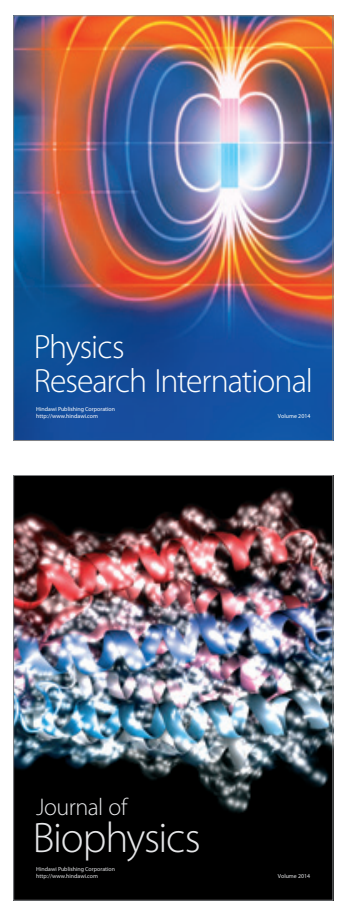
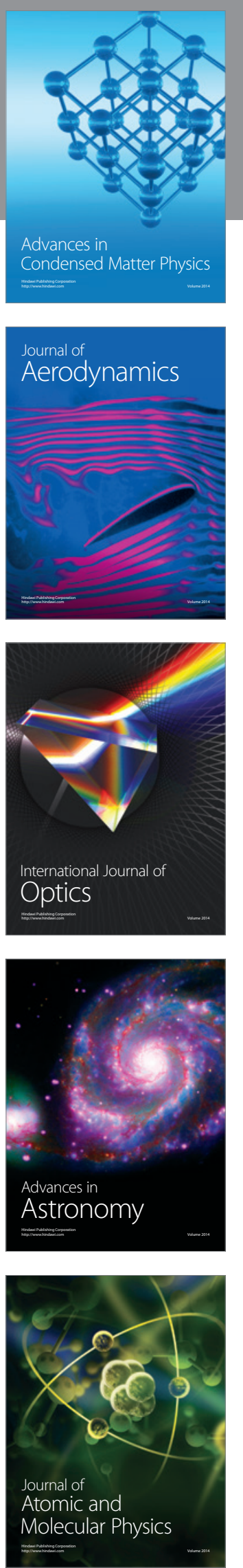